\title{
Late Quaternary sea level and environmental changes from relic carbonate deposits of the western margin of India
}

\author{
V Purnachandra Rao*, G Rajagopalan**, K H Vora* and F Almeida* \\ * National Institute of Oceanography, Dona Paula, Goa 403 004, India. \\ ** Birbal Sahni Institute of Palaeobotany, 53 University Road, Lucknow, India.
}

\begin{abstract}
Relic carbonate deposits along the western margin of India occur as dolomite crusts, aragonite sands (pelletal / oolitic) and aragonite-cemented limestones, oyster shells, corals, encrusted coralline algal and foraminiferal-dominated nodules. The petrology and mineralogy of the deposits indicate that except for aragonite sands and foraminiferal nodules, the others were formed in shallow marine conditions and serve as sea level indicators. Radiocarbon dates were measured for 62 relic deposits covering the entire margin. The age of these deposits on the continental shelf off Cape Comorin and Mangalore, between 110 and $18 \mathrm{~m}$ depth, ranges between $12,610{ }^{14} \mathrm{C}$ yr $\mathrm{BP}$ and $6,390{ }^{14} \mathrm{C}$ yr $\mathrm{BP}$. On the northwestern margin of India, especially on the carbonate platform (between 64 and $100 \mathrm{~m}$ ), the age ranges from 17,250 to $6,730{ }^{14} \mathrm{C}$ yr BP. The relic deposits of the Gulf of Kachchh at depths between 35 and $25 \mathrm{~m}$ are dated at $12,550-9,630{ }^{14} \mathrm{C}$ yr BP. The age vs. depth plot of the relic deposits further indicates that the Gulf of Kachchh was inundated much early, atleast by 15 ka, after the Last Glacial Maximum, and was subjected to uplift and subsidence during the Holocene. The carbonate platform subsided during the early Holocene. Some of the relic deposits between Cape Comorin and Mangalore plot on or, closely follow the glacio-eustatic sea level curve. Despite abundant siliciclastic flux discharged by the Narmada and Tapti during the early Holocene, the platform off these rivers is largely devoid of this flux and carbonate sedimentation continued until $6,700{ }^{14} \mathrm{C}$ yr BP. We suggest that the river-derived sediment flux diverted southwards under the influence of the SW monsoon current and, thereby, increased the turbidity on the shelf and slope southeast of the carbonate platform and facilitated the formation of deeper water foraminiferal nodules off Vengurla-Goa.
\end{abstract}

\section{Introduction}

Relic shallow water sediments are exposed on the outer continental shelf of the western margin of India, between the Gulf of Kachchh and Cape Comorin (Nair and Pylee 1968; von Stackelberg 1972). A carbonate platform, also known as Fifty Fathom Flat, extending 4 degrees of latitude, occurs on the outer continental shelf of the northwestern margin of India (figure 1). As a consequence, the width of the relic zone is more in the northwestern part and becomes narrow towards the southwestern margin of India. Several investigators studied the geomorphic features and associ- ated sediments on the outer shelf off western India (Nair 1975; Nair et al 1979; Vora and Almeida 1990; Wagle et al 1994; Rao et al 1994; Rao and Veerayya 1996; Rao et al 1996; Vora et al 1996; Rao and Wagle 1997). The studies reveal the existence of reefal structures along the shelf break and biohermal structures on the carbonate platform. So far, relic deposits from 20 stations of the carbonate platform and off Vengurla-Goa were dated by radiocarbon methods (Nair 1974; Nair et al 1979; Vora and Almeida 1990; Rao et al 1994; Rao and Veerayya 1996). Using a few of these dates, Kale and Rajaguru (1985) and Hashimi et al (1995) attempted to construct Holocene sea level curves

Keywords. Late Quaternary sea level; western margin of India; subsidence; uplift; depositional environment. 


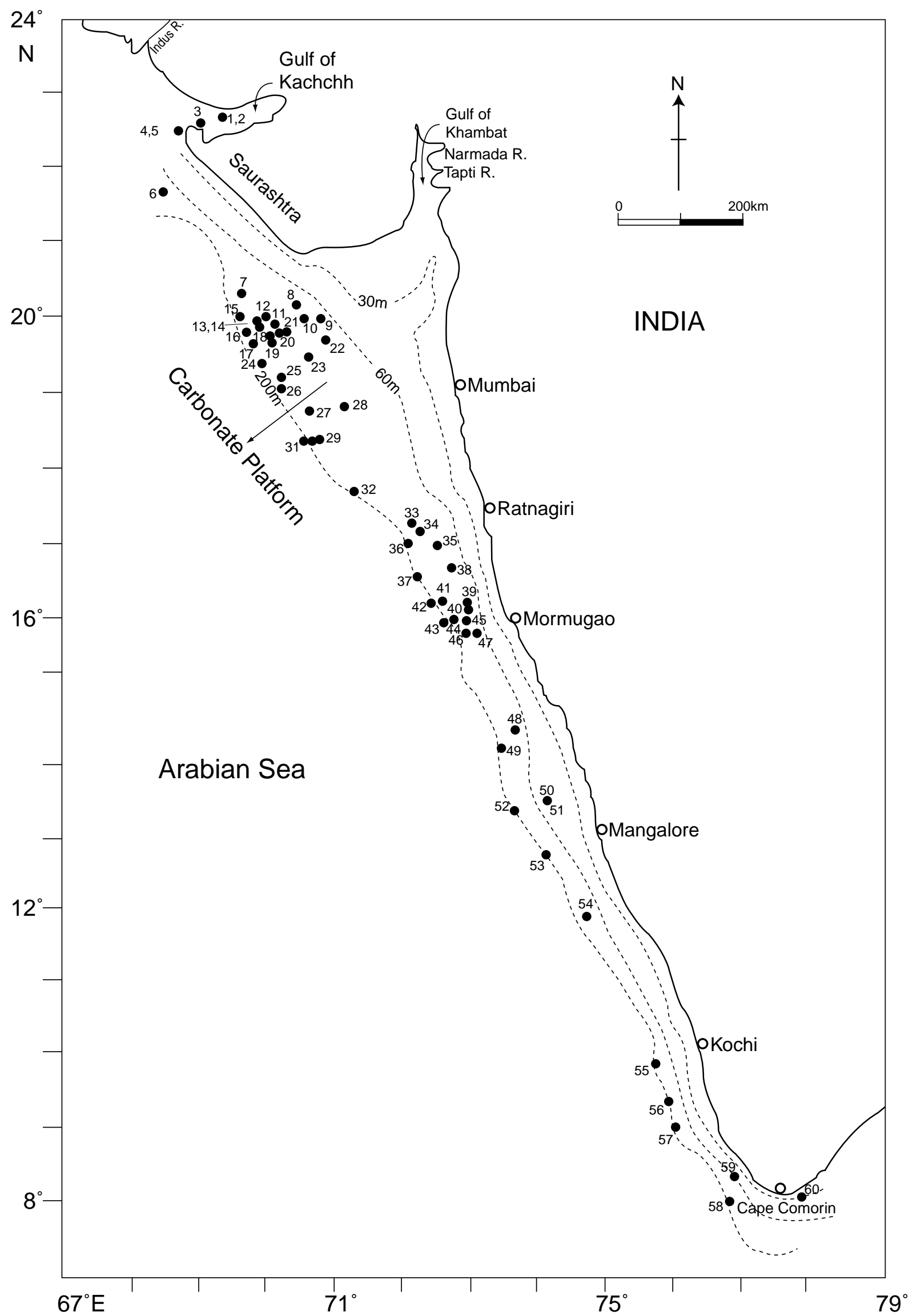

Figure 1. Location of the samples along the western margin of India used for petrology, mineralogy and radiocarbon measurements. The numbers correspond to serial number in tables 1 and 2 . 
for the western margin of India. These two curves are indeed different from one another and also differ with the glacio-eustatic sea level curve of Fairbanks (1989). The data points making the sea level curves in Kale and Rajaguru (1985) and Hashimi et al (1995) are largely inferred/estimated ages and, actual measured dates of the samples fall away from the curves. Rao et al (1996) have discussed several problems in the data used for preparing these curves and also explained why the curves are unacceptable to represent sea level changes off western India. Moreover, no radiocarbon dates were available for the relic reefs/sediments of the larger part of the western Indian margin (off GoaCape Comorin, Saurashtra or the Gulf of Kachchh) when these sea level curves were constructed. In other words, the significance of these relic sediments in relation to late Quaternary sea level and environmental changes has not yet been comprehended. Here, we have determined the age of as many as 42 relic deposits of different types covering the entire margin under the Project "Sea level changes along the western margin of India during the late Quaternary" funded by the Department of Science and Technology, New Delhi. The purpose of the paper is to update the inventory of the radiocarbon ages of relic deposits on this margin, synthesize results on sea level changes in relation to glacio-eustatic sea level and impact of sea level/environmental changes on sediment depositional environment during the late Quaternary.

\section{Material and methods}

The sediments/sedimentary rocks recovered from the western margin of India during different cruises of $R V$ Gaveshani (G), ORV Sagar Kanya (SK) and $M V$ Nand Rachit (NR) by Peterson Grab. Pipe dredge with chain bag at the bottom and bucket dredge were used for the present study (figure 1). Table 1 shows the locations, depths and description of the sediments and also the samples chosen for different analyses. In the case of dredge samples the location and depth of the samples are estimates of their averages at the start and end of dredging. Samples selected from each station were ultrasonically cleaned. The samples were split into two halves. One half was used for preparing thin sections and studied under petrological microscope. The other half was powdered, subjected to X-ray diffraction studies and then dating by radiocarbon methods were carried out at the Birbal Sahni Institute of Palaeobotony, Lucknow. Minerals present in each sample and ratio of major carbonate minerals are shown in table 1 . Dolomite (D), aragonite (A) and high-magnesium calcite $(\mathrm{H})$ are marine minerals; their ratios indicate relative abundance of one mineral with respect to the other. Since low-magnesium calcite $(\mathrm{C})$ can originate from the influence of meteoric water, one should be cautious if its percentage is higher in a given sample. We are aware that the calcite and aragonite have different absorption coefficients for X-rays and peak heights of these minerals in the X-ray diffractogram are not a true reflection of their abundance. We have prepared a calibration curve based on pure mixtures of aragonite $(A)$ and calcite $(C)$. From this curve it is evident that the weight percentage of aragonite is $80 \%, 70 \%, 60 \%$ and $50 \%$ when $\mathrm{A} / \mathrm{C}$ peak height ratio in the mixture is 1.0 , $0.5,0.3$ and 0.18, respectively. Samples analysed here have consistently shown higher $\mathrm{A} / \mathrm{C}$ ratios $(>2)$ (see table 1) indicating that the weight percentage of aragonite is $>90 \%$ and therefore the radiocarbon age dating on them is reliable. Oyster shells (see table 1) are originally made up of lowmagnesium calcite $(\mathrm{C})$ and the ${ }^{14} \mathrm{C}$ date on them is valid. Measured ${ }^{14} \mathrm{C}$ ages were calibrated using CALIB rev. 4.3 of Stuiver et al (1998) and are given in table 2. During calibration a local deviation in $\Delta \mathrm{R}-100 \pm 30$ for Goa-Cape Comorin region and $163 \pm 30$ for Gulf of Kachchh - Goa was used, following Dutta et al (2001). Corrected radiocarbon ages are obtained by subtracting surface ocean reservoir age -400 years and 100 years in local deviation in $\Delta R$. We have discussed the corrected and calibrated radiocarbon ages in the text so that one can compare these ages with the age of the sediments on land. Three sediment samples and a dolomite crust were dated by Accelerator Mass Spectrometer (AMS) at the Woods Hole Oceanographic Institution (WHOI), USA. The conventional radiocarbon ages are younger than that of AMS ages by 500 to 800 years for the samples of the same station (see table 2). This arose because AMS age measurements were made on a few oolite grains carefully picked up from $>250 \mu \mathrm{m}$ fraction, whereas conventional radiocarbon age determinations were done on the sediments in the size range $250-850 \mu \mathrm{m}$.

\section{Results}

\subsection{Relic deposits of the Gulf of Kachchh}

The type of relic deposits recovered along the continental margin varied significantly from north to south (table 1). The samples recovered at different stations from the Gulf of Kachchh are large sheet limestones encrusted by 0.5 to $1.0 \mathrm{~cm}$ thick dolomite crusts, $3 \mathrm{~cm}$ sized lense-shaped dolomite crusts, corals belonging to the Faviidae family (Leptastrea transversa) and micritic limestones (table 1; figure 2a). Dolomite crusts 


\begin{tabular}{|c|c|c|c|c|c|c|c|c|c|c|c|c|c|c|c|c|c|c|c|}
\hline 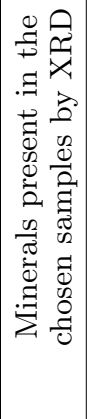 & 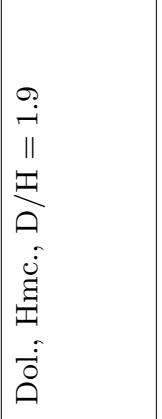 & 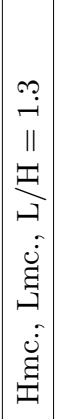 & 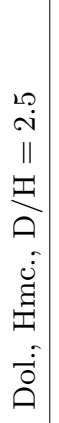 & 氙 & 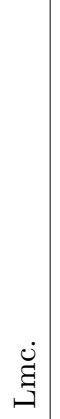 & 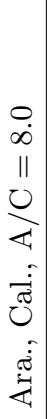 & 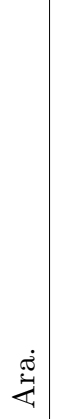 & 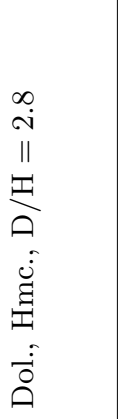 & 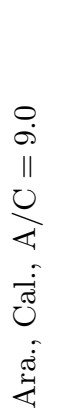 & & 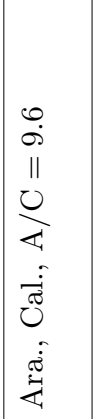 & 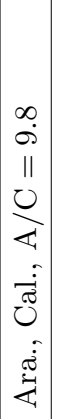 & 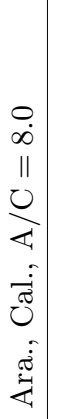 & 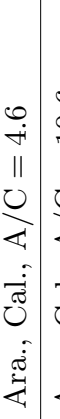 & 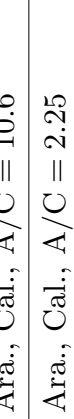 & 荌 & 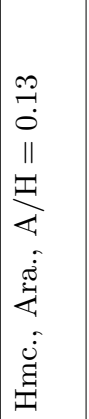 & 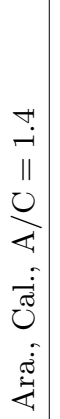 & 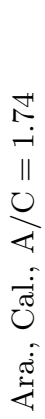 \\
\hline 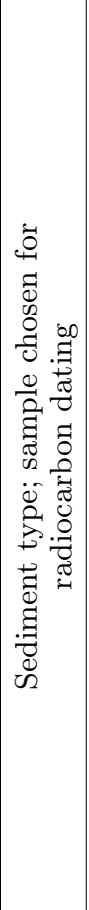 & 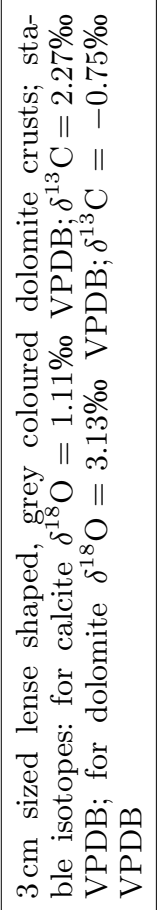 & 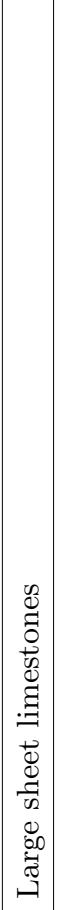 & 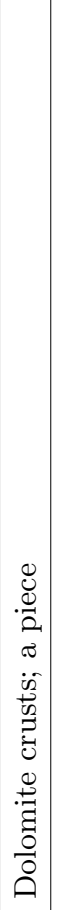 & 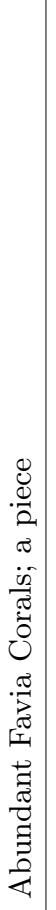 & 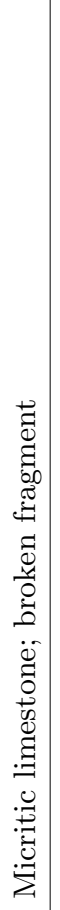 & 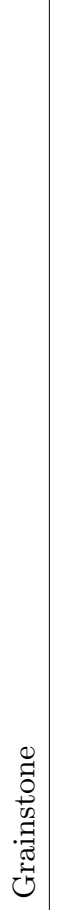 & 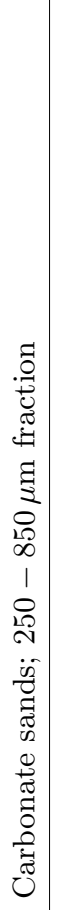 & 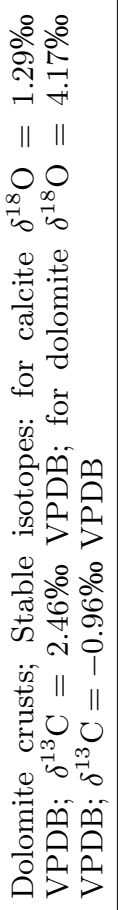 & 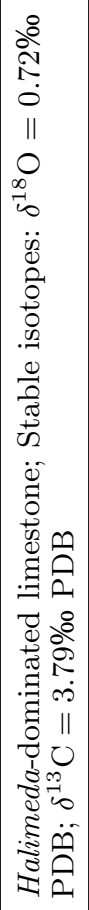 & 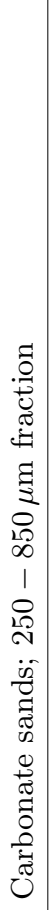 & 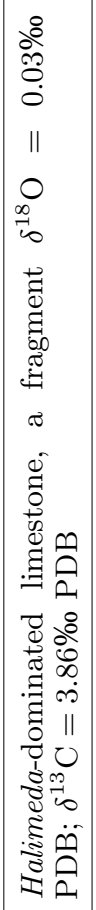 & 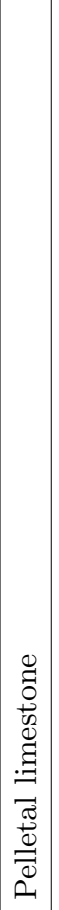 & 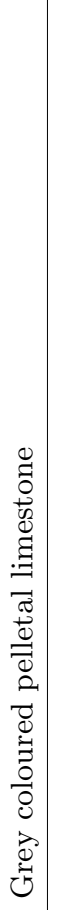 & 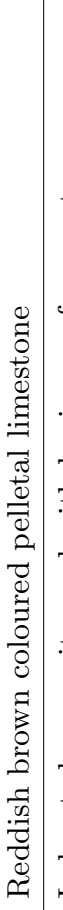 & 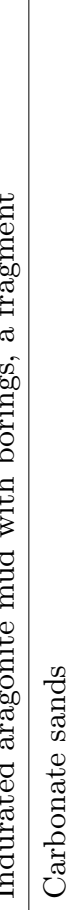 & 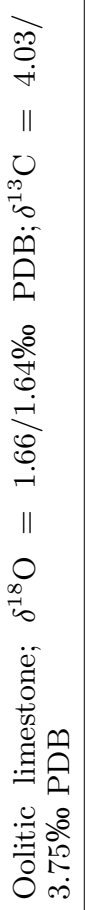 & 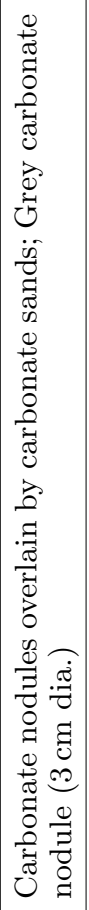 & 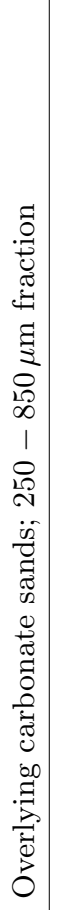 & 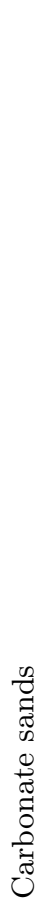 \\
\hline 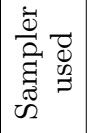 & $\dot{\theta}$ & $\begin{array}{c}\dot{A} \\
\dot{n}\end{array}$ & $\begin{array}{l}\dot{\theta} \\
\dot{n}\end{array}$ & $\begin{array}{l}\dot{a} \\
\dot{a}\end{array}$ & \begin{tabular}{|}
$\dot{\theta}$ \\
$\dot{n}$
\end{tabular} & $\begin{array}{l}\dot{\theta} \\
\dot{a}\end{array}$ & $\begin{array}{l}\dot{0} \\
\dot{ن}\end{array}$ & 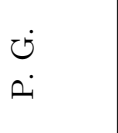 & $\dot{\theta}$ & $\begin{array}{l}\dot{ن} \\
\dot{0}\end{array}$ & $\dot{\theta}$ & $\begin{array}{c}\dot{\theta} \\
\dot{n}\end{array}$ & $\begin{array}{l}\dot{a} \\
\dot{a}\end{array}$ & $\begin{array}{ll}\dot{\theta} & 0 \\
\dot{n} & \end{array}$ & \begin{tabular}{l|l} 
\\
$\dot{0}$
\end{tabular} & $\begin{array}{l}\dot{0} \\
\text { نं }\end{array}$ & $\begin{array}{l}ن \dot{~} \\
\text { i. }\end{array}$ & $\begin{array}{l}\dot{0} \\
\dot{ن}\end{array}$ & $\begin{array}{l}\dot{0} \\
\text { نَ }\end{array}$ \\
\hline 营 & 8 & 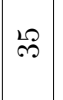 & $\stackrel{2}{N}$ & $\stackrel{\infty}{\sim}$ & $\stackrel{\infty}{\infty}$ & $\stackrel{\mathcal{\Xi}}{\exists}$ & $\stackrel{120}{\infty}$ & tే & 18 & $\infty$ & 208 & 20 & $\infty$ & $\triangleright$ & 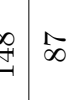 & $\stackrel{9}{9}$ & $\not \infty$ & $\vec{\infty}$ & $\vec{\infty}$ \\
\hline 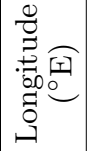 & 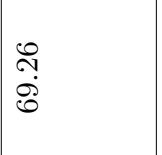 & $\mid \begin{array}{c}0 \\
\sim \\
\infty \\
0 \\
0\end{array}$ & $\begin{array}{l}\stackrel{8}{0} \\
\dot{0}\end{array}$ & $\begin{array}{l}F \\
\dot{0} \\
0\end{array}$ & $\mid \begin{array}{l}7 \\
\rightarrow \\
0 \\
0\end{array}$ & $\begin{array}{l}0 \\
\stackrel{1}{0} \\
0 \\
0\end{array}$ & $\begin{array}{l}\stackrel{\mathscr{H}}{\sharp} \\
\stackrel{0}{0}\end{array}$ & 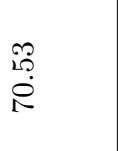 & 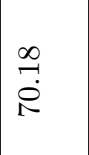 & 点 & $\underset{\stackrel{9}{+}}{\stackrel{9}{8}}$ & $\mid \begin{array}{l}20 \\
20 \\
98 \\
0\end{array}$ & $\begin{array}{l}12 \\
100 \\
98 \\
0\end{array}$ & $\begin{array}{l}2: 0 \\
10 \\
0 \\
0\end{array}$ & 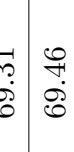 & $\begin{array}{l}\text { 前 } \\
\text { Oे }\end{array}$ & $\frac{9}{\stackrel{2}{R}}$ & $\underset{\vec{R}}{\stackrel{2}{R}}$ & $\stackrel{9}{\vec{R}}$ \\
\hline 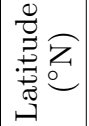 & $\begin{array}{l}\stackrel{O}{4} \\
\text { ล̀ }\end{array}$ & $\mid \begin{array}{l}\text { I } \\
\stackrel{\text { N}}{\text { N }}\end{array}$ & $\begin{array}{l}0 \\
\stackrel{\oplus}{0} \\
\stackrel{N}{\mathrm{~N}}\end{array}$ & $\begin{array}{c}\overrightarrow{\widetilde{p}} \\
\stackrel{\mathrm{s}}{\mathrm{s}}\end{array}$ & 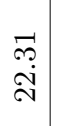 & $\begin{array}{l}\vec{\infty} \\
\overrightarrow{\mathrm{N}}\end{array}$ & $\begin{array}{l}\stackrel{\infty}{0} \\
\stackrel{\sim}{\sim}\end{array}$ & $\begin{array}{l}\stackrel{8}{\circ} \\
\stackrel{10}{\rightarrow}\end{array}$ & $\begin{array}{l}\text { Fै } \\
\stackrel{9}{9}\end{array}$ & $\begin{array}{l}\infty \\
1 \\
\stackrel{2}{9} \\
\stackrel{-}{-1}\end{array}$ & $\begin{array}{l}1 \\
10 \\
0 \\
9 \\
-9\end{array}$ & $\begin{array}{l}1 \\
20 \\
9 \\
9 \\
-9\end{array}$ & $\begin{array}{l}\text { 占 } \\
\stackrel{10}{9}\end{array}$ & 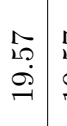 & 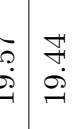 & $\begin{array}{l}\stackrel{L}{\circ} \\
\stackrel{9}{9} \\
\stackrel{9}{9}\end{array}$ & 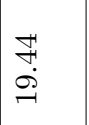 & $\begin{array}{l}\underset{+}{+} \\
\stackrel{9}{\rightarrow}\end{array}$ & $\stackrel{\Re}{\stackrel{?}{\mathscr{G}}}$ \\
\hline 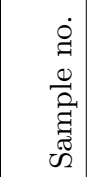 & 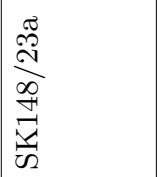 & 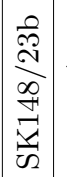 & 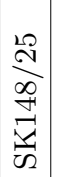 & 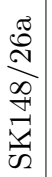 & 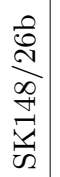 & 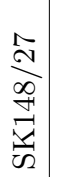 & $\underset{\partial}{\stackrel{d}{\partial}}$ & 疍 & 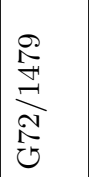 & 3 & 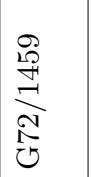 & 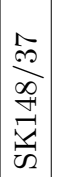 & 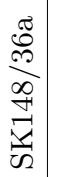 & 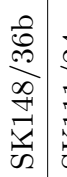 & 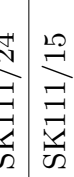 & $\mathbb{Z}$ & $\stackrel{0}{\stackrel{0}{\sigma}}$ & 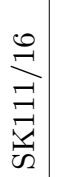 & $\underset{⿱ 乛}{\vec{\Xi}}$ \\
\hline$\stackrel{9}{9}$ & -1 & $|\sim|$ & $\infty$ & $\mid$ & 10 & | & 1 & $\infty$ & 0 & & & $|\stackrel{\sim}{\sim}|$ & $\stackrel{m}{\rightarrow}$ & $\exists \mid$ & 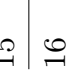 & & & & \\
\hline
\end{tabular}




\begin{tabular}{|c|c|c|c|c|c|c|c|c|c|c|c|c|c|c|c|c|c|c|c|c|c|c|c|}
\hline 究 & 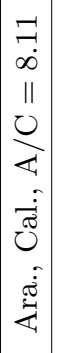 & & & 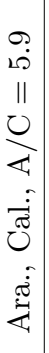 & & & 究 & 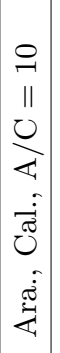 & 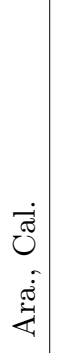 & 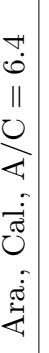 & 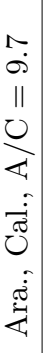 & 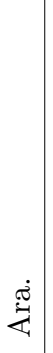 & 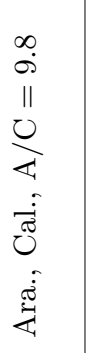 & 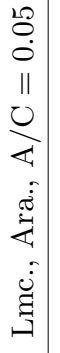 & 总 & : & & 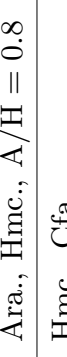 & & 离 & 弟 & & \\
\hline 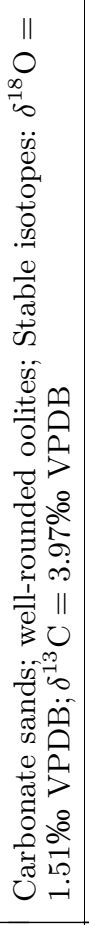 & 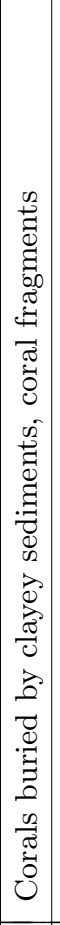 & 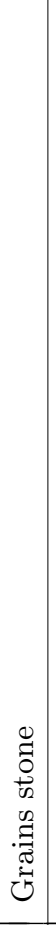 & 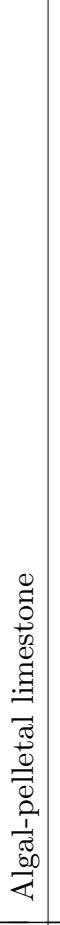 & 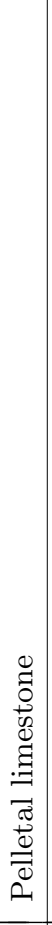 & 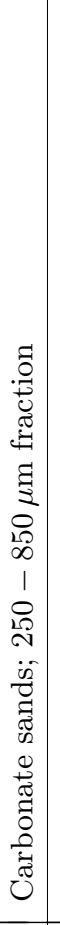 & 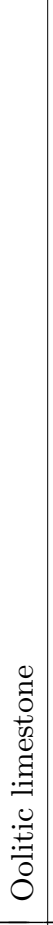 & 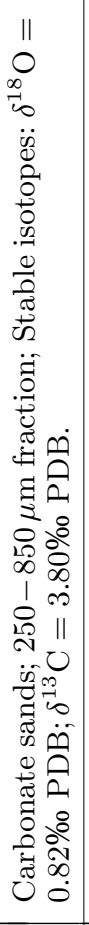 & 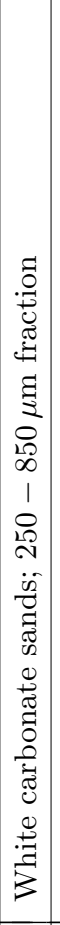 & 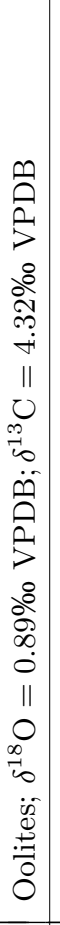 & 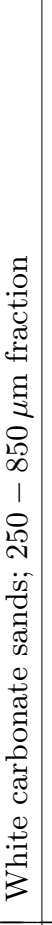 & $\frac{d}{i}$ & 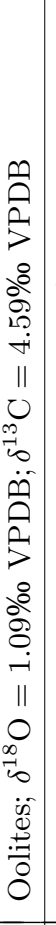 & 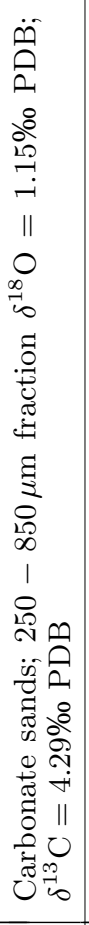 & 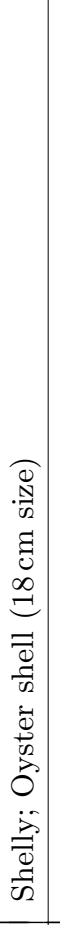 & 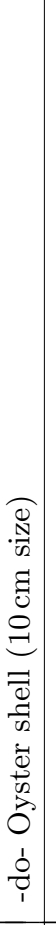 & $\begin{array}{l}\dot{c} \\
\\
1\end{array}$ & 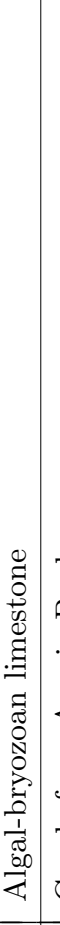 & 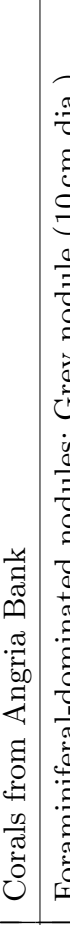 & 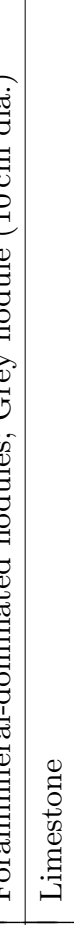 & 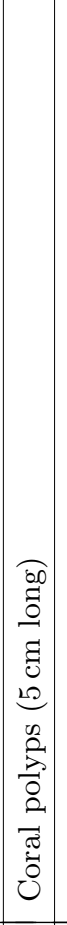 & 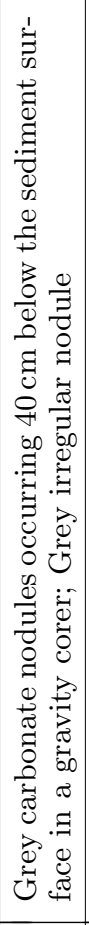 & 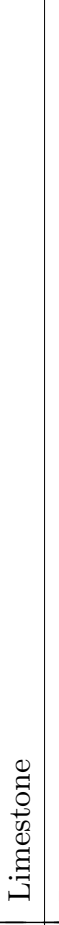 & 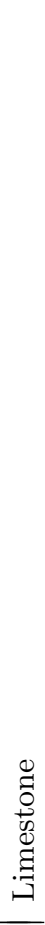 \\
\hline U & $\mid \begin{array}{c}\dot{0} \\
\dot{2}\end{array}$ & $\begin{array}{c}\dot{A} \\
\dot{\Omega}\end{array}$ & $\begin{array}{c}\dot{A} \\
\dot{a}\end{array}$ & $\begin{array}{c}\dot{A} \\
\dot{n}\end{array}$ & $\begin{array}{l}\dot{ن} \\
ن\end{array}$ & $\begin{array}{l}\dot{ن} \\
\dot{ن}\end{array}$ & $\begin{array}{l}\text { نं } \\
\text { ن }\end{array}$ & $\mid \begin{array}{l}\dot{\sigma} \\
\dot{2}\end{array}$ & 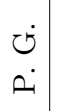 & $\begin{array}{l}\dot{0} \\
\text { : }\end{array}$ & $\begin{array}{l}\dot{ن} \\
\text { i }\end{array}$ & $\begin{array}{l}\dot{\delta} \\
\dot{ن}\end{array}$ & $\begin{array}{l}\dot{0} \\
\text { Di }\end{array}$ & $\begin{array}{l}\dot{\varphi} \\
\dot{\varphi}\end{array}$ & $\begin{array}{l}\dot{\theta} \\
\dot{\varphi}\end{array}$ & $\begin{array}{l}\dot{\varphi} \\
\dot{\varphi}\end{array}$ & $\begin{array}{l}\dot{\theta} \\
\dot{0}\end{array}$ & 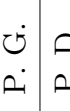 & 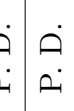 & $\begin{array}{c}\dot{A} \\
\dot{n}\end{array}$ & نَ & $\begin{array}{l}\dot{\theta} \\
\dot{D}\end{array}$ & $\dot{\theta}$ \\
\hline$\stackrel{\infty}{\infty}$ & $\mid \begin{array}{l}\infty \\
0\end{array}$ & $\stackrel{\infty}{\infty}$ & $\stackrel{8}{\stackrel{8}{\rightarrow}}$ & $\vec{b}$ & $\underset{\sim}{\stackrel{ }{\prime}}$ & $\stackrel{\infty}{\circ}$ & $\triangleright$ & $\mid \begin{array}{l}\infty \\
\infty\end{array}$ & 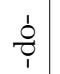 & $\sigma$ & 당 & $\frac{\dot{\theta}}{\vec{p}}$ & $\underset{-}{\stackrel{-}{1}}$ & 8 & $\stackrel{\infty}{\infty}$ & $R$ & $\underset{\infty}{\infty}$ & ๙ & 8 & $\ddot{\circ}$ & i & ঃ & ธิ \\
\hline 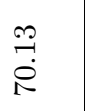 & $\mid \begin{array}{c}\vec{b} \\
\dot{i}\end{array}$ & $\begin{array}{l}\infty \\
\stackrel{\infty}{2} \\
\stackrel{R}{R}\end{array}$ & $\begin{array}{c}0 \\
100 \\
9 \\
0\end{array}$ & $\begin{array}{l}\stackrel{0}{1} \\
\stackrel{1}{R}\end{array}$ & $\begin{array}{l}10 \\
\stackrel{2}{0} \\
\stackrel{1}{R}\end{array}$ & $\begin{array}{l}\stackrel{P}{1} \\
\stackrel{R}{R}\end{array}$ & $\begin{array}{l}\stackrel{20}{ت} \\
\stackrel{1}{i}\end{array}$ & 缹 & $\frac{1}{0}$ & $\begin{array}{l}\stackrel{g}{F} \\
\stackrel{R}{R}\end{array}$ & $\begin{array}{l}\text { fे } \\
\stackrel{8}{8}\end{array}$ & $\begin{array}{l}\dot{d} \\
\frac{8}{1}\end{array}$ & $\underset{\stackrel{\overbrace{}}{i}}{\stackrel{i}{i}}$ & $\underset{\stackrel{\sim}{i}}{\stackrel{i}{i}}$ & $\begin{array}{c}\overrightarrow{\mathrm{N}} \\
\stackrel{\mathrm{N}}{\mathrm{i}}\end{array}$ & $\begin{array}{l}\stackrel{P}{4} \\
i \\
i\end{array}$ & \begin{tabular}{l|l}
$\stackrel{2}{0}$ \\
$\stackrel{1}{\mathrm{i}}$
\end{tabular} & 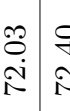 & 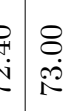 & $\begin{array}{l}\overrightarrow{0} \\
\stackrel{i}{i}\end{array}$ & 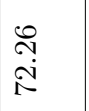 & 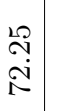 & $\underset{\stackrel{\Re}{\stackrel{P}{i}}}{\stackrel{\sim}{i}}$ \\
\hline F & $\begin{array}{l}\vec{H} \\
\stackrel{\leftrightarrow}{\rightarrow}\end{array}$ & 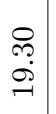 & $\begin{array}{l}\infty \\
\stackrel{2}{-} \\
\stackrel{9}{\rightarrow}\end{array}$ & $\begin{array}{l}\stackrel{8}{0} \\
\stackrel{9}{\rightarrow}\end{array}$ & $\begin{array}{l}8 \\
\stackrel{9}{9}\end{array}$ & $\begin{array}{l}\stackrel{P}{+} \\
\stackrel{\infty}{\rightarrow}\end{array}$ & $\begin{array}{l}\stackrel{\infty}{\rightarrow} \\
\infty \\
\sim\end{array}$ & $\begin{array}{l}\underset{-}{-1} \\
\infty \\
\rightarrow\end{array}$ & $\begin{array}{l}\dot{1} \\
\stackrel{1}{1}\end{array}$ & 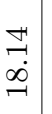 & $\begin{array}{l}\underset{\mathbb{1}}{\infty} \\
\stackrel{\infty}{-}\end{array}$ & $\begin{array}{l}\dot{0} \\
\\
\\
1\end{array}$ & 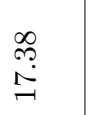 & $\begin{array}{l}\stackrel{ }{二} \\
\stackrel{ }{二}\end{array}$ & 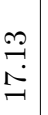 & 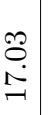 & 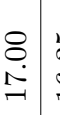 & 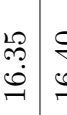 & 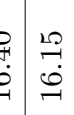 & 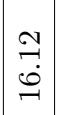 & 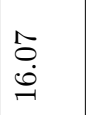 & $\begin{array}{l}10 \\
\stackrel{-1}{6} \\
-9\end{array}$ & $\begin{array}{l}\stackrel{8}{0} \\
\stackrel{0}{-1}\end{array}$ \\
\hline$\vec{\Xi}$ & 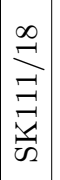 & $\grave{\Sigma}$ & 尔 & 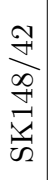 & $\frac{0}{z_{z}}$ & Fै & 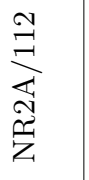 & 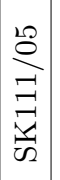 & $\begin{array}{l}{ }^{*}{ }_{1} \\
\\
\\
1\end{array}$ & 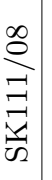 & 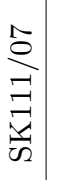 & $\begin{array}{l}* \\
\vdots \\
0 \\
\\
1 \\
1\end{array}$ & 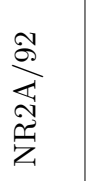 & $\begin{array}{l}\vec{\infty} \\
\underset{\mathbb{d}}{\infty} \\
\stackrel{\vec{z}}{z}\end{array}$ & 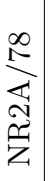 & 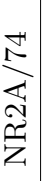 & $\begin{array}{l}\text { 岱 } \\
y^{2}\end{array}$ & 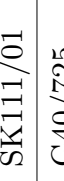 & 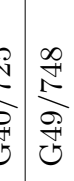 & 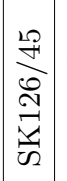 & 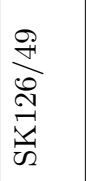 & 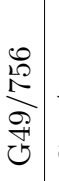 & 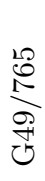 \\
\hline & ฐ̃ & & & & & $\stackrel{N}{\sim}$ & & & & & & & & & & & & & & & & & \\
\hline
\end{tabular}




\begin{tabular}{|c|c|c|c|c|c|c|c|c|c|c|c|c|c|c|c|c|c|c|}
\hline 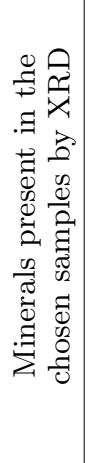 & 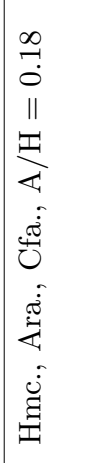 & 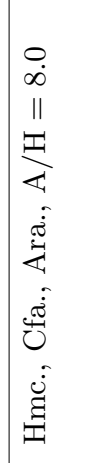 & 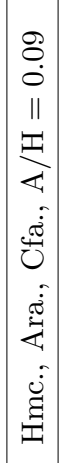 & 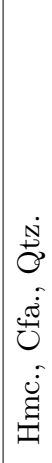 & & 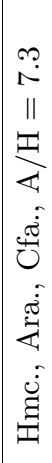 & 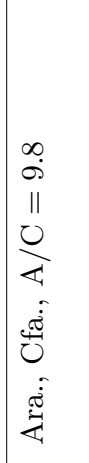 & 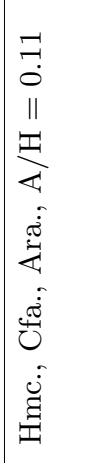 & 要 & 晃 & 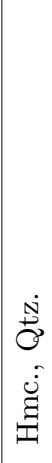 & 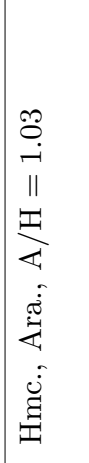 & 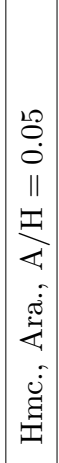 & 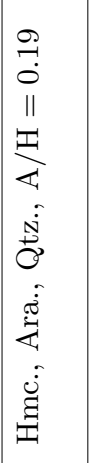 & 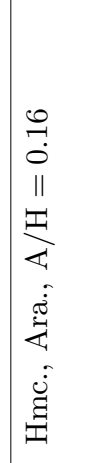 & 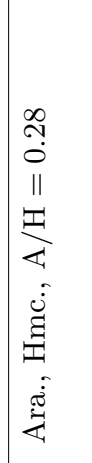 & 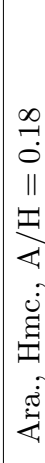 & 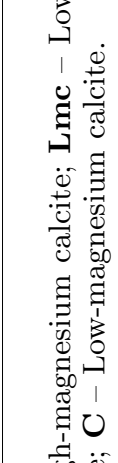 \\
\hline 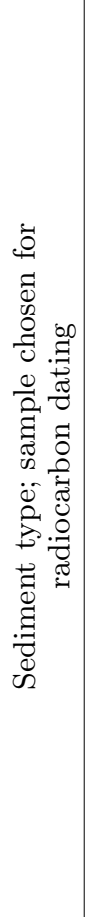 & 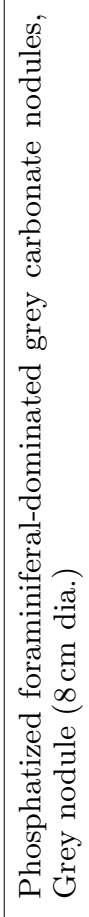 & 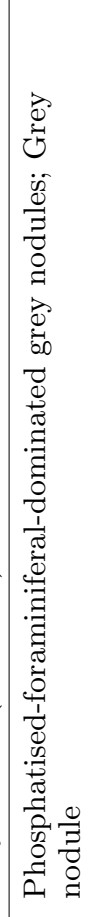 & $\frac{1}{1}$ & $\frac{1}{i}$ & 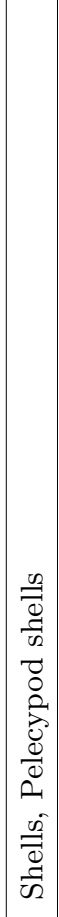 & 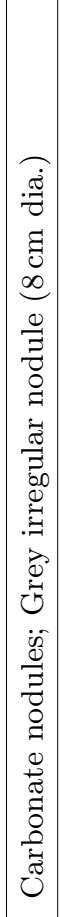 & 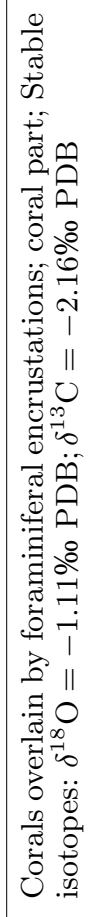 & 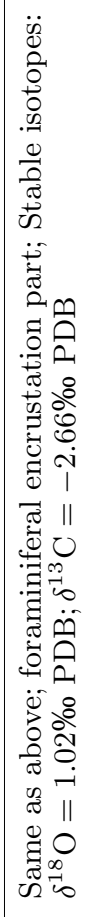 & 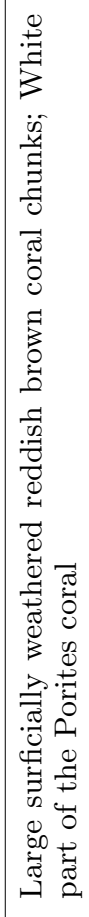 & 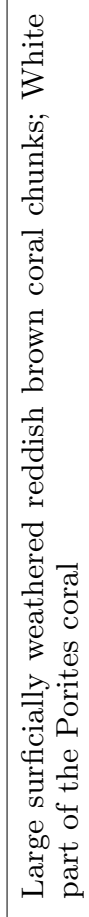 & 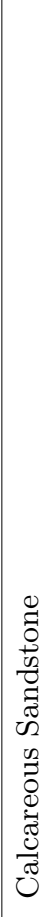 & 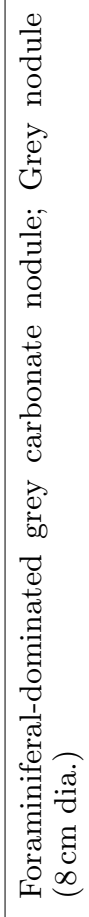 & 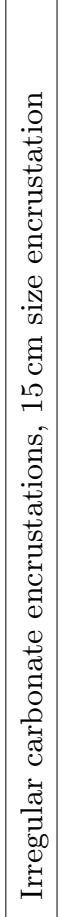 & 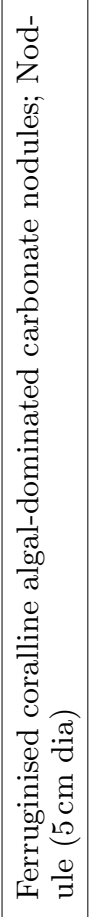 & 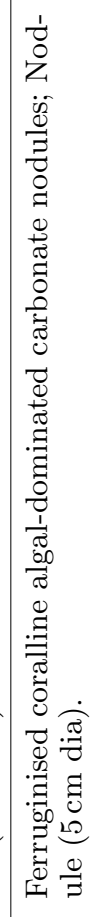 & 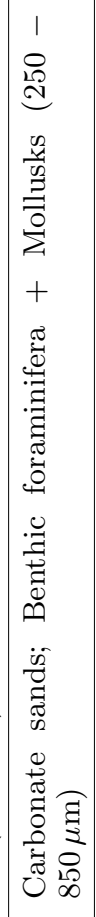 & i⿱ & 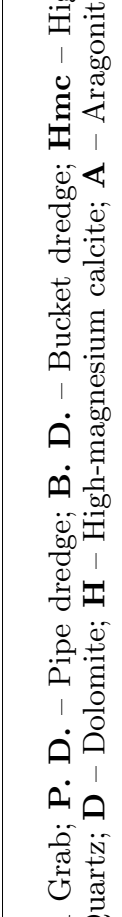 \\
\hline 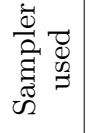 & $\dot{\rho}$ & $\dot{\theta} \vec{\theta}$ & $\begin{array}{l}\dot{\theta} \\
\dot{\varphi}\end{array}$ & $\dot{\rho}$ & $\begin{array}{l}\dot{a} \\
\dot{D}\end{array}$ & $\begin{array}{l}\dot{\theta} \\
\dot{a}\end{array}$ & $\dot{\theta}$ & $\dot{\theta}$ & A & 0 & $\dot{\theta}$ & $\dot{\theta}$ & $\begin{array}{l}\dot{\theta} \\
\dot{\theta}\end{array}$ & $\dot{\theta}$ & $\dot{A}$ & $\begin{array}{l}\dot{0} \\
\text { نص }\end{array}$ & $\begin{array}{l}\dot{0} \\
\text { í }\end{array}$ & \\
\hline 苛 & 28 & ৪ & 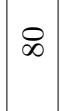 & $\stackrel{\infty}{0}$ & $\begin{array}{l}\infty \\
10\end{array}$ & $\mathscr{8}$ & $R$ & R & $\stackrel{20}{9}$ & $\stackrel{ }{\exists}$ & $\varnothing$ & $\stackrel{\infty}{\infty}$ & $\begin{array}{l}\infty \\
\infty\end{array}$ & $\stackrel{ }{\circ}$ & 8 & F & $\stackrel{\infty}{\sim}$ & \\
\hline 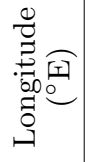 & $\begin{array}{l}\mathcal{N} \\
\stackrel{1}{\mathcal{B}} \\
\mathcal{i}\end{array}$ & $\begin{array}{l}\overrightarrow{0} \\
\dot{p}\end{array}$ & 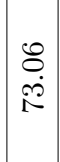 & 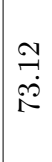 & $\mid \begin{array}{l}\stackrel{+}{r} \\
\stackrel{\rho}{R}\end{array}$ & $\mid \begin{array}{l}\hat{i} \\
\stackrel{i}{i} \\
\hat{i}\end{array}$ & 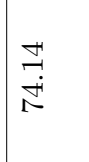 & 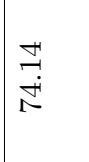 & 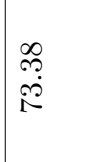 & $\kappa$ & $\infty$ & 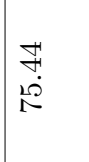 & 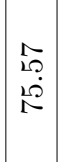 & $\begin{array}{l}\overrightarrow{0} \\
\dot{0} \\
\hat{0}\end{array}$ & 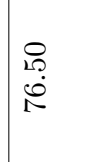 & 兽 & 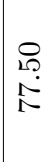 & \\
\hline 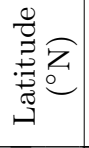 & 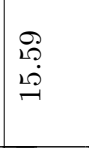 & 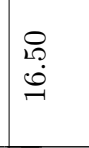 & 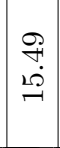 & 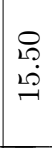 & 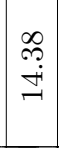 & $\begin{array}{l}\mathcal{N} \\
\mathcal{I} \\
\dot{J}\end{array}$ & $\begin{array}{l}\stackrel{\rho}{0} \\
\stackrel{9}{9}\end{array}$ & 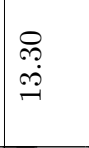 & ๙ิ) & $\begin{array}{l}\text { ㄱ } \\
\text { S } \\
\text { I }\end{array}$ & $\stackrel{\infty}{\stackrel{\infty}{=}}$ & $\begin{array}{l}\overrightarrow{7} \\
\dot{8}\end{array}$ & $\begin{array}{l}\overrightarrow{1} \\
\dot{8}\end{array}$ & $\begin{array}{l}12 \\
20 \\
0 \\
0 \\
0\end{array}$ & 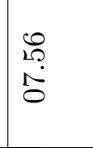 & $\begin{array}{l}\stackrel{8}{1} \\
\ddot{0}\end{array}$ & $\begin{array}{l}\mathcal{1} \\
\infty \\
0 \\
0\end{array}$ & \\
\hline 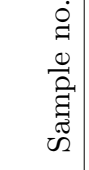 & 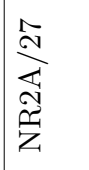 & 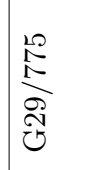 & 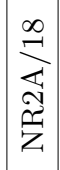 & $\frac{N}{\frac{N}{2}}$ & 0 & 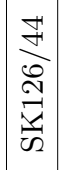 & 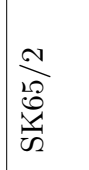 & 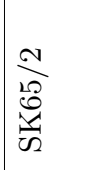 & 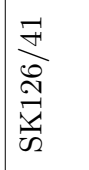 & 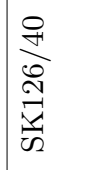 & 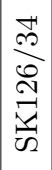 & 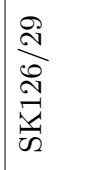 & 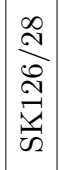 & 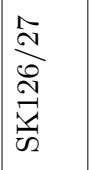 & 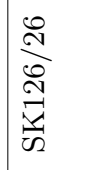 & 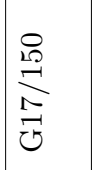 & 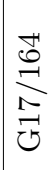 & \\
\hline & & & & & $\mid \underset{\forall}{\infty}$ & & 180 & & & & & & $\left|\begin{array}{l}\mid \\
10\end{array}\right|$ & & & & & \\
\hline
\end{tabular}




\begin{tabular}{|c|c|c|c|c|c|c|c|c|c|c|c|c|c|c|c|c|c|c|c|c|c|}
\hline 苞 & 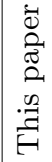 & 守 & 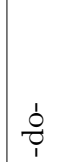 & 官 & 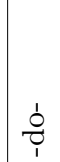 & 定 & 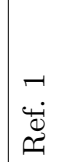 & 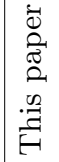 & $\begin{array}{l}\sim \\
\ddot{\mathbb{U}} \\
\tilde{\sim}\end{array}$ & 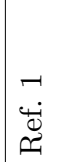 & $\begin{array}{l}\text { N } \\
\stackrel{\mathbb{W}}{*}\end{array}$ & 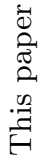 & 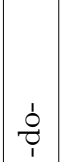 & $\frac{1}{0}$ & 家 & $\frac{1}{0}$ & 蛋 & 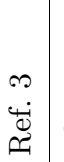 & 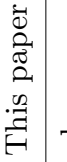 & 㒸 & 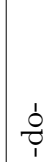 \\
\hline 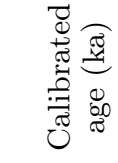 & 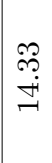 & 1 & 笭 & $\begin{array}{l}\vec{\infty} \\
\infty \\
\stackrel{0}{-}\end{array}$ & 1 & 1 & $\begin{array}{l}\stackrel{2}{2} \\
\stackrel{9}{9} \\
\stackrel{9}{9}\end{array}$ & 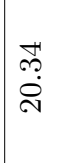 & $\begin{array}{l}\vec{\infty} \\
\dot{\sigma}\end{array}$ & $\begin{array}{l}0 \\
\stackrel{1}{0} \\
\stackrel{9}{1}\end{array}$ & $\begin{array}{l}\qquad 8 \\
\infty \\
\infty \\
\infty\end{array}$ & 궁 & $\mid \begin{array}{c}\infty \\
\stackrel{N}{0} \\
\stackrel{0}{\sim}\end{array}$ & $\mid \begin{array}{c}\stackrel{\leftrightarrow}{\mathscr{g}} \\
\stackrel{\circ}{\sim}\end{array}$ & 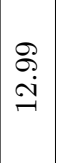 & 足 & $\begin{array}{l}\stackrel{20}{\rightarrow} \\
\stackrel{9}{9}\end{array}$ & $\begin{array}{l}\stackrel{\infty}{\sim} \\
\stackrel{n}{9} \\
\stackrel{9}{9}\end{array}$ & 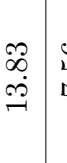 & & $\underset{\infty}{\stackrel{+}{\infty}}$ \\
\hline 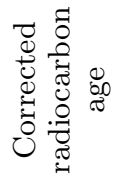 & 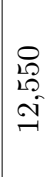 & 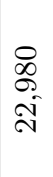 & $\begin{array}{l}0 \\
0 \\
0 \\
0 \\
0 \\
-1\end{array}$ & $\begin{array}{l}0 \\
\mathscr{0} \\
0 \\
0 \\
0\end{array}$ & & $\begin{array}{l}0 \\
2 \\
\infty \\
0 \\
0 \\
0\end{array}$ & $\begin{array}{l}8 \\
\vdots \\
\vdots \\
\infty\end{array}$ & $\begin{array}{l}0 \\
\stackrel{0}{A} \\
\stackrel{N}{N} \\
=\end{array}$ & $\begin{array}{l}2 \\
2 \\
10 \\
\infty \\
\infty\end{array}$ & $\begin{array}{l}8 \\
\stackrel{8}{1} \\
0\end{array}$ & $\begin{array}{l}\text { R } \\
\text { s. } \\
\text { N }\end{array}$ & $\begin{array}{l}\stackrel{8}{9} \\
\stackrel{5}{\circ}\end{array}$ & $\mid \begin{array}{l}\infty \\
\infty \\
\approx \\
\infty\end{array}$ & $\mid \begin{array}{l}\mathscr{8} \\
\mathscr{E} \\
0 \\
0\end{array}$ & $\begin{array}{l}\stackrel{0}{9} \\
\exists \\
=\end{array}$ & $\begin{array}{l}8 \\
\stackrel{8}{0} \\
1\end{array}$ & 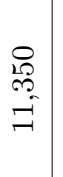 & $\begin{array}{l}\infty \\
\stackrel{\infty}{+} \\
=\end{array}$ & $\begin{array}{c}\stackrel{8}{0} \\
\mathcal{I}\end{array}$ & 总 & 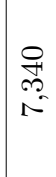 \\
\hline 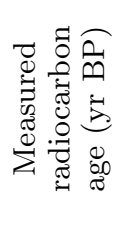 & $\begin{array}{l}8 \\
8 \\
+1 \\
0 \\
0 \\
0 \\
0 \\
9 \\
9\end{array}$ & 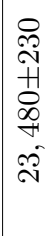 & $\begin{array}{l}8 \\
0 \\
+1 \\
+ \\
0 \\
0 \\
-1 \\
=\end{array}$ & 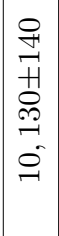 & $\begin{array}{l}8 \\
8 \\
0 \\
9 \\
1\end{array}$ & 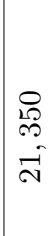 & $\begin{array}{l}8 \\
8 \\
0 \\
+1 \\
8 \\
\vdots \\
0 \\
0 \\
0\end{array}$ & $\begin{array}{l}2 \\
\infty \\
\infty \\
1 \\
0 \\
1 \\
1 \\
1 \\
-1 \\
-1\end{array}$ & $\begin{array}{l}8 \\
\infty \\
-H \\
10 \\
\infty \\
N \\
0 \\
0\end{array}$ & $\begin{array}{l}8 \\
0 \\
+1 \\
0 \\
8 \\
8 \\
0 \\
0\end{array}$ & 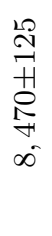 & 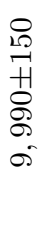 & $\begin{array}{c}\stackrel{0}{0} \\
\stackrel{1}{1} \\
+1 \\
0 \\
0 \\
0 \\
0\end{array}$ & 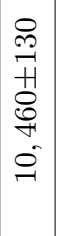 & $\begin{array}{l}: 0 \\
0 \\
+1 \\
0 \\
0 \\
0 \\
= \\
=\end{array}$ & \begin{tabular}{l|}
8 \\
0 \\
$\stackrel{1}{1}$ \\
8 \\
8 \\
0 \\
1 \\
1
\end{tabular} & 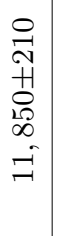 & $\begin{array}{l}2 \\
\infty \\
0 \\
+1 \\
0 \\
0 \\
0 \\
= \\
=\end{array}$ & 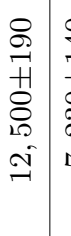 & 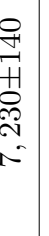 & $\begin{array}{l}8 \\
0 \\
+H \\
+1 \\
0 \\
\infty \\
1 \\
1\end{array}$ \\
\hline 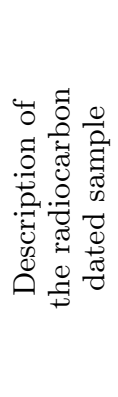 & $\begin{array}{l}\frac{n}{20} \\
\frac{0}{0} \\
0 \\
0 \\
0 \\
.0 \\
0 \\
0\end{array}$ & 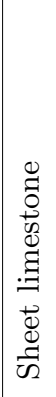 & 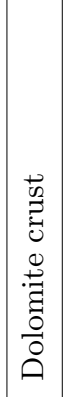 & $\begin{array}{l}\tilde{\pi} \\
\tilde{\delta} \\
0\end{array}$ & 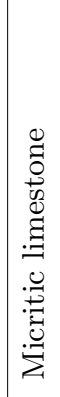 & 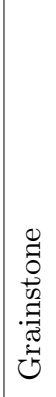 & 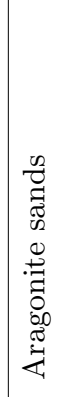 & 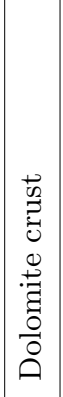 & 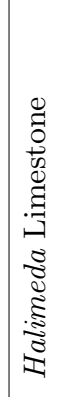 & 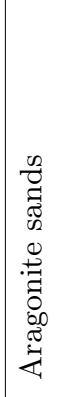 & 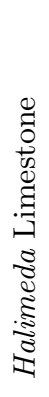 & 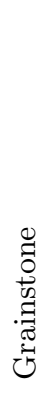 & 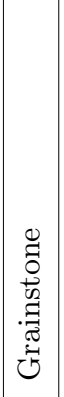 & 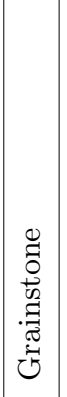 & 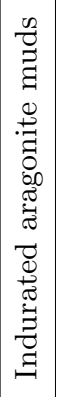 & 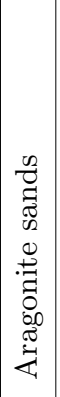 & 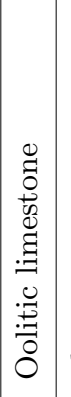 & di & 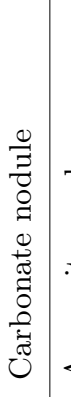 & 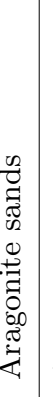 & 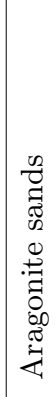 \\
\hline 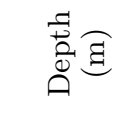 & 8 & $\stackrel{20}{60}$ & $\stackrel{20}{\mathrm{~N}}$ & $\stackrel{\infty}{\sim}$ & $\stackrel{\infty}{\sim}$ & $\stackrel{\mathcal{I}}{=}$ & $\stackrel{10}{\infty}$ & రే & 10 & $\infty$ & 28 & $\stackrel{20}{12}$ & $\infty$ & $\infty$ & $\stackrel{\infty}{\rightarrow}$ & $\infty$ & $\stackrel{9}{9}$ & คి & $\dot{\infty}_{\infty}$ & $\vec{\infty}$ & $\vec{\infty}$ \\
\hline 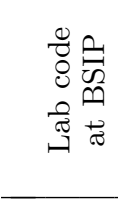 & 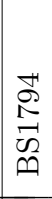 & 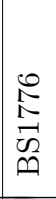 & 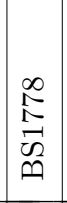 & $\begin{array}{l}\Omega \\
R \\
N \\
\sqrt[N]{n} \\
n\end{array}$ & $\begin{array}{l}\vec{D} \\
D \\
\vec{N} \\
\infty \\
\infty\end{array}$ & $\begin{array}{l}\stackrel{\mathscr{N}}{\stackrel{2}{2}} \\
\tilde{L}\end{array}$ & 1 & 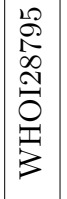 & 1 & 1 & 1 & $\begin{array}{l}\mathscr{D} \\
\infty \\
0 \\
\vec{n} \\
n\end{array}$ & $\begin{array}{l}0 \\
0 \\
0 \\
\tilde{n} \\
\tilde{n} \\
\mid\end{array}$ & $\mid \begin{array}{l}\tilde{D} \\
\infty \\
\tilde{D} \\
\tilde{D} \\
\tilde{n}\end{array}$ & $\mid \begin{array}{l}\infty \\
0 \\
m \\
\vec{m} \\
\tilde{\omega} \\
m\end{array}$ & $\begin{array}{l}\vec{D} \\
\vec{W} \\
\vec{n} \\
\\
\end{array}$ & 1 & 1 & 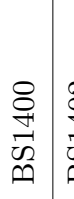 & & 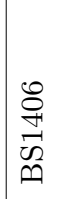 \\
\hline$\ddot{\sim \Omega}$ & 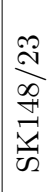 & 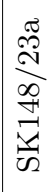 & 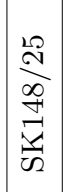 & 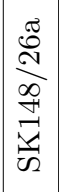 & 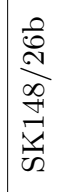 & 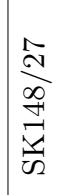 & $\stackrel{\vec{D}}{\stackrel{2}{O}}$ & 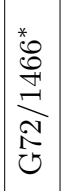 & 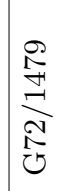 & $\stackrel{\infty}{\stackrel{\infty}{\rho}}$ & 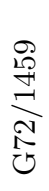 & 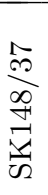 & 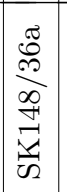 & 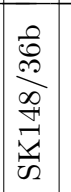 & 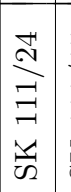 & 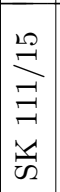 & & $\underset{F}{F}$ & 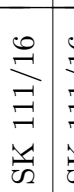 & 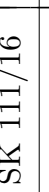 & 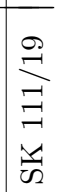 \\
\hline & & & & $r$ & 100 & & N & $\infty$ & 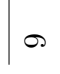 & $\stackrel{9}{2}$ & & $\tilde{F} 1$ & 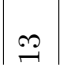 & $\exists$ & 129 & $\stackrel{0}{\mathscr{2}}$ & 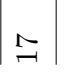 & & $\stackrel{\infty}{\rightarrow}$ & 9 & ๙ิ \\
\hline
\end{tabular}




\begin{tabular}{|c|c|c|c|c|c|c|c|c|c|c|c|c|c|c|c|c|c|c|c|}
\hline 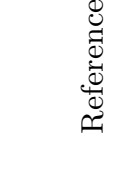 & $\frac{1}{7}$ & 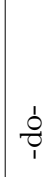 & î & 岕 & $\begin{array}{l}\vec{i} \\
\dot{\vec{d}} \\
\stackrel{x}{\mid c}\end{array}$ & 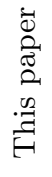 & 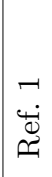 & $\begin{array}{l}\overrightarrow{-} \\
\dot{\mathbb{v}} \\
\sim\end{array}$ & 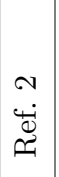 & 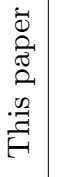 & 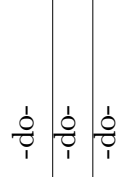 & 竎 & $\begin{array}{l}\sim \\
\dot{\mathbb{U}} \\
\sim\end{array}$ & 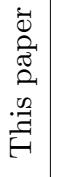 & i⿱ & $\overbrace{1}^{1}$ & 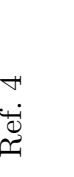 & 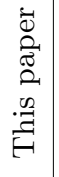 & ته \\
\hline 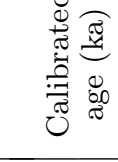 & $\begin{array}{l}\stackrel{\Im}{\sharp} \\
\stackrel{9}{9}\end{array}$ & 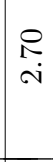 & $\begin{array}{l}\vec{N} \\
\dot{\sigma}\end{array}$ & $\begin{array}{l}\stackrel{\infty}{+} \\
\stackrel{\leftrightarrow}{\sim}\end{array}$ & $\mid$ & 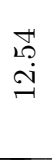 & $\begin{array}{l}\stackrel{N}{N} \\
\stackrel{N}{I}\end{array}$ & $\begin{array}{l}R \\
0 \\
0\end{array}$ & $\mid \begin{array}{l}\overrightarrow{0} \\
\infty \\
\infty\end{array}$ & $\begin{array}{l}\vec{\sigma} \\
\dot{\sigma}\end{array}$ & 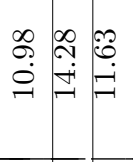 & \begin{tabular}{|l|}
$\overrightarrow{2}$ \\
$\stackrel{a}{i}$ \\
$\vec{I}$
\end{tabular} & $\begin{array}{l}\mathfrak{N} \\
\stackrel{1}{0} \\
\stackrel{\ddots}{0}\end{array}$ & \begin{tabular}{l}
$\infty$ \\
\multirow{2}{*}{} \\
$\stackrel{-}{-1}$
\end{tabular} & $\begin{array}{l}\vec{\infty} \\
\stackrel{\infty}{\rightarrow}\end{array}$ & 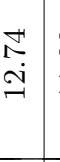 & ت & 1 & $\begin{array}{l}\infty \\
\infty \\
\infty\end{array}$ \\
\hline 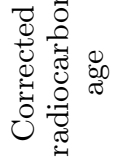 & $\begin{array}{l}0 \\
\infty \\
\infty \\
= \\
=\end{array}$ & 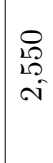 & 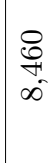 & $\begin{array}{l}0 \\
0 \\
0 \\
0 \\
9\end{array}$ & $\mid \begin{array}{l}0 \\
1 \\
10 \\
0 \\
-1 \\
-1\end{array}$ & $\begin{array}{l}8 \\
10 \\
0 \\
0 \\
-1\end{array}$ & $\begin{array}{l}0 \\
0 \\
0 \\
0 \\
0 \\
=\end{array}$ & $\begin{array}{l}8 \\
R \\
\infty\end{array}$ & $\begin{array}{l}\stackrel{9}{1} \\
\infty \\
1 \\
1\end{array}$ & $\begin{array}{l}\stackrel{8}{\circ} \\
\infty \\
\infty\end{array}$ & 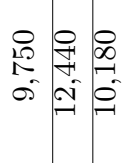 & $\begin{array}{l}8 \\
0 \\
0 \\
0 \\
-1\end{array}$ & $\begin{array}{l}8 \\
\infty \\
1 \\
0 \\
0\end{array}$ & $\begin{array}{l}\underset{8}{1} \\
\text { on } \\
\text { s. }\end{array}$ & 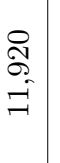 & 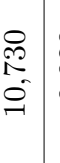 & $\begin{array}{l}\text { बू. } \\
\text { s. } \\
\infty\end{array}$ & 1 & 宓 \\
\hline 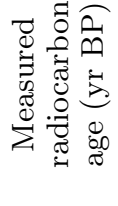 & 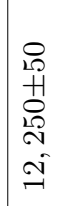 & $\begin{array}{l}8 \\
0 \\
+1 \\
0 \\
0 \\
0 \\
0 \\
\infty\end{array}$ & 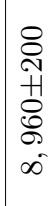 & 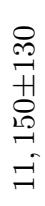 & 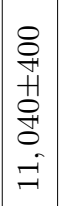 & 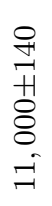 & $\begin{array}{l}0 \\
0 \\
0 \\
+1 \\
0 \\
0 \\
0 \\
0 \\
-1 \\
=1\end{array}$ & 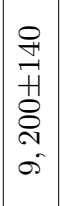 & $\mid$\begin{tabular}{c|}
2 \\
$\infty$ \\
0 \\
+1 \\
0 \\
0 \\
$\infty$ \\
$\infty$ \\
$\infty$
\end{tabular} & 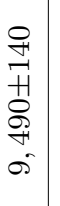 & 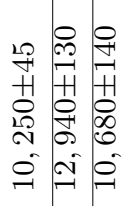 & $\begin{array}{l}\stackrel{2}{+} \\
+4 \\
+ \\
8 \\
= \\
= \\
= \\
=\end{array}$ & $\begin{array}{l}0 \\
\stackrel{2}{0} \\
+ \\
+ \\
\infty \\
0 \\
0 \\
0 \\
-1\end{array}$ & $\begin{array}{l}0 \\
\exists \\
\text { H. } \\
8 \\
\stackrel{1}{0} \\
0\end{array}$ & 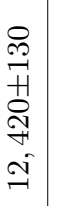 & 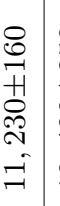 & 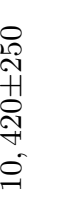 & 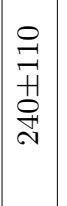 & 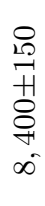 \\
\hline 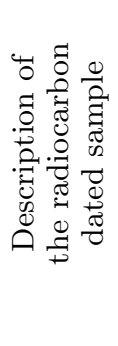 & 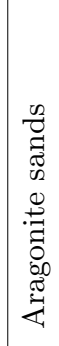 & రై & 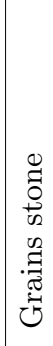 & 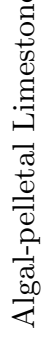 & do & 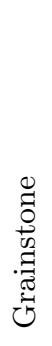 & 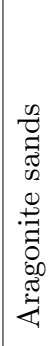 & 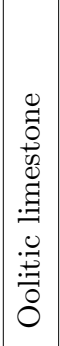 & 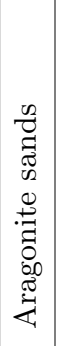 & 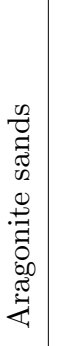 & 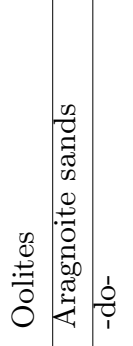 & 辛 & 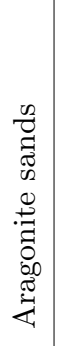 & 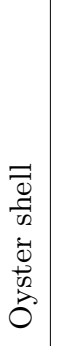 & $\frac{1}{\substack{1\\
}}$ & $\overbrace{1}^{1}$ & 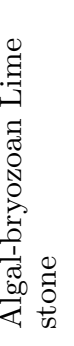 & 宽 & 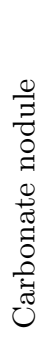 \\
\hline 葶 & $\stackrel{\infty}{\infty}$ & $\infty$ & $\infty$ & 点 & $\begin{array}{l}80 \\
9\end{array}$ & ठే & $\underset{-1}{8}$ & $\begin{array}{c}\infty \\
\infty \\
\infty\end{array}$ & $\infty$ & $\infty$ & $\begin{array}{l}\infty \\
\infty\end{array}$ & $\mid \begin{array}{l}1 \\
0 \\
1\end{array}$ & 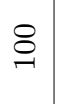 & 8 & 18 & R & $\underset{\infty}{\infty}$ & Nิ & $\infty$ \\
\hline 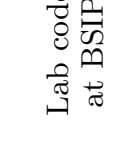 & 恿 & 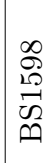 & I & I & & $\underset{\infty}{\infty}$ & I & I & $\begin{array}{l}\mathcal{F} \\
\overrightarrow{7} \\
\dot{5}\end{array}$ & 点 & 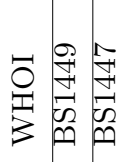 & 官 & 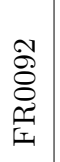 & 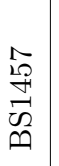 & 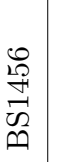 & 吾 & & 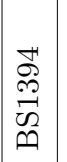 & 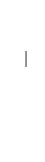 \\
\hline 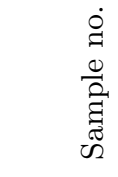 & $\stackrel{\stackrel{*}{N}}{\vec{\exists}}$ & 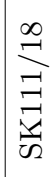 & $\stackrel{0}{\Sigma}$ & 감 & $\begin{aligned} \mathrm{F} \\
\text { F }\end{aligned}$ & 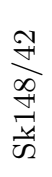 & $\stackrel{9}{\mathrm{z}}$ & F & 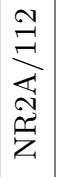 & 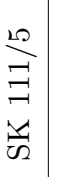 & 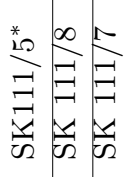 & 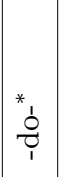 & 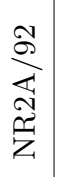 & 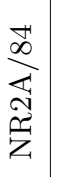 & 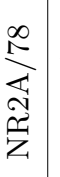 & 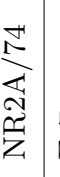 & 1 & $\mid \begin{array}{l}\vec{J} \\
\exists \\
\exists \\
i \\
c\end{array}$ & $\begin{array}{l}\frac{1}{N} \\
\text { Â } \\
\text { Oै }\end{array}$ \\
\hline & 5 & & & & & & $\stackrel{N}{N}$ & s & $|\stackrel{\infty}{\infty}|$ & $\sigma$ & 危 & $\vec{\omega}$ & $\approx$ & תֶ, & ले & חמ & & $\infty$ & \\
\hline
\end{tabular}




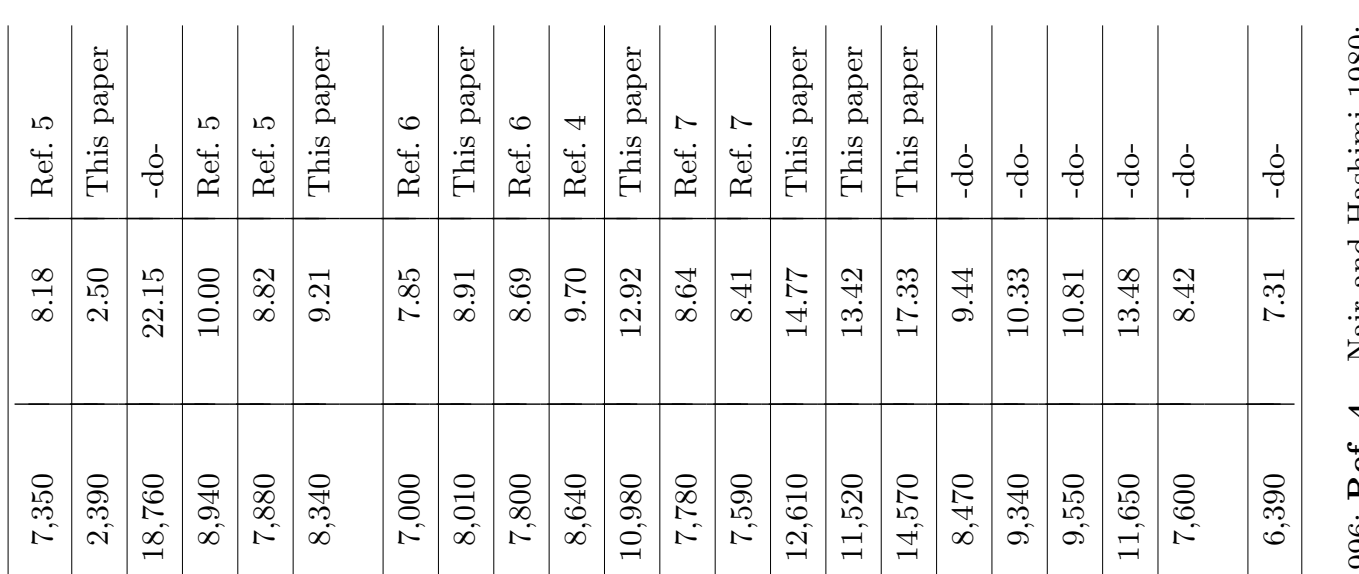

$\ddot{\infty}$

贯

चี

西

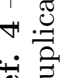

五

$\ddot{\circ} \theta$

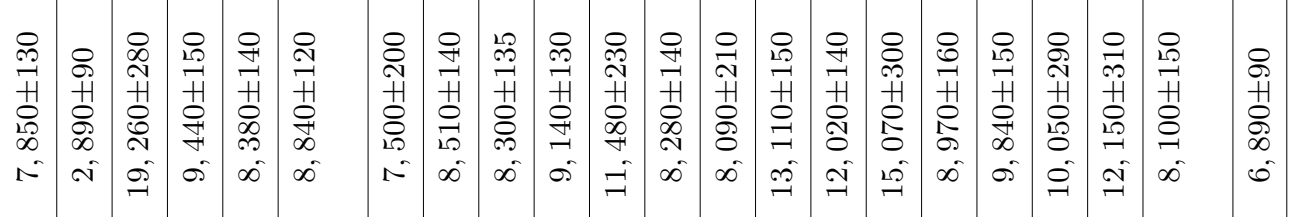

蛋

त्ञ

콕

ส

雪

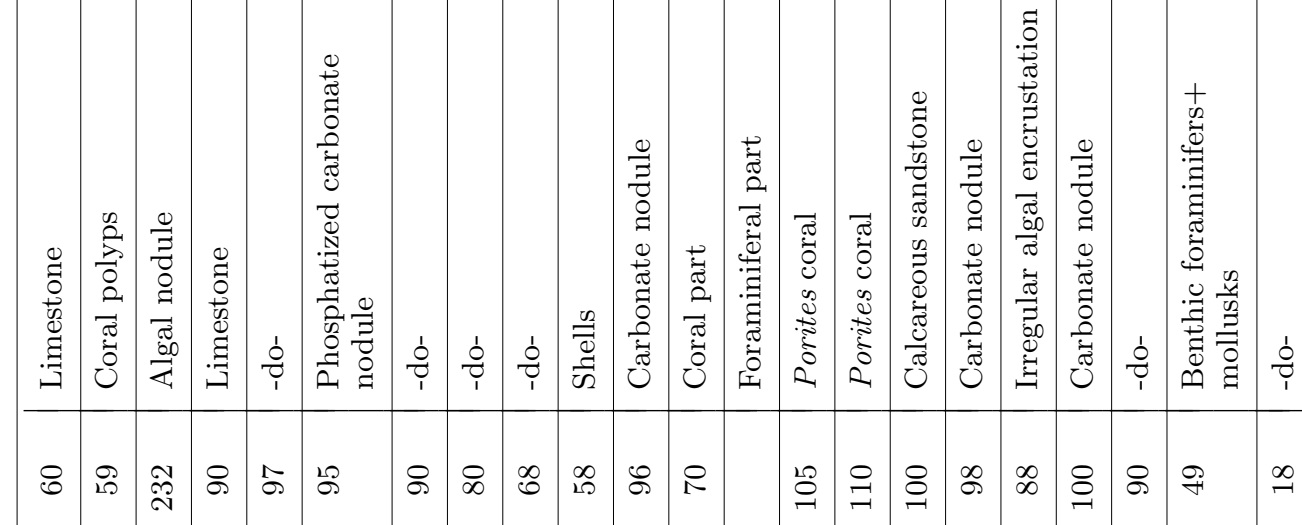

\begin{tabular}{|c|c|c|c|c|c|c|c|c|c|c|c|c|c|c|c|c|c|c|c|c|}
\hline 1 & $\begin{array}{l}0 \\
\infty \\
\cong \\
\ddot{2} \\
\tilde{n}\end{array}$ & 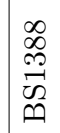 & 1 & 1 & 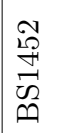 & & $\mid \begin{array}{l}\overrightarrow{2} \\
\overrightarrow{1} \\
\vec{n} \\
m\end{array}$ & 1 & 1 & $\begin{array}{l}20 \\
\infty \\
\\
\vec{\sim} \\
\tilde{n}\end{array}$ & 1 & & $\begin{array}{l}\vec{\infty} \\
\stackrel{0}{9} \\
\vec{\sim} \\
\tilde{n}\end{array}$ & $\begin{array}{l}\vec{\infty} \\
\stackrel{2}{2} \\
\overrightarrow{2} \\
\ddot{m}\end{array}$ & & 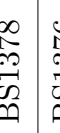 & 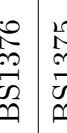 & 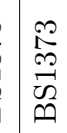 & $\begin{array}{l}8 \\
0 \\
\overrightarrow{0} \\
\tilde{n} \\
m\end{array}$ & 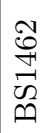 \\
\hline 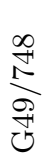 & 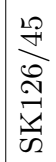 & 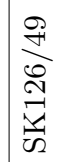 & 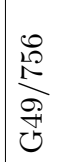 & 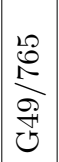 & 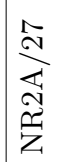 & 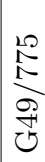 & 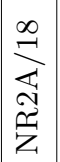 & 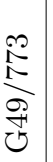 & 量 & 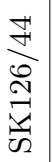 & 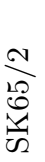 & $\begin{array}{l}\frac{v}{d:} \\
0 \\
v \\
v 2\end{array}$ & 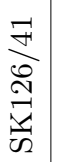 & 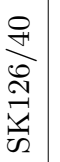 & 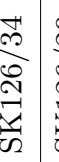 & 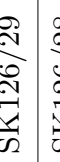 & 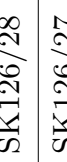 & 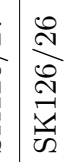 & 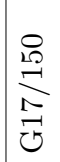 & $\frac{\overrightarrow{0}}{\stackrel{\Delta}{-}}$ \\
\hline$\stackrel{\sigma}{\circ}$ & 우 & $F$ & $\underset{F}{\mathcal{F}}$ & $\stackrel{\Re}{\leftrightarrow}$ & $\not{F}$ & L & $\mathscr{\mathscr { H }}$ & 각 & $\stackrel{\infty}{\leftrightarrow}$ & $\stackrel{ㄱ}{7}$ & $\stackrel{\circ}{\circ}$ & $\sqrt{10}$ & ㄱำ & ஜ & 20 & 80 & $\begin{array}{ll}0 \\
0\end{array}$ & $\begin{array}{l}\infty \\
10\end{array}$ & is & $b$ \\
\hline
\end{tabular}




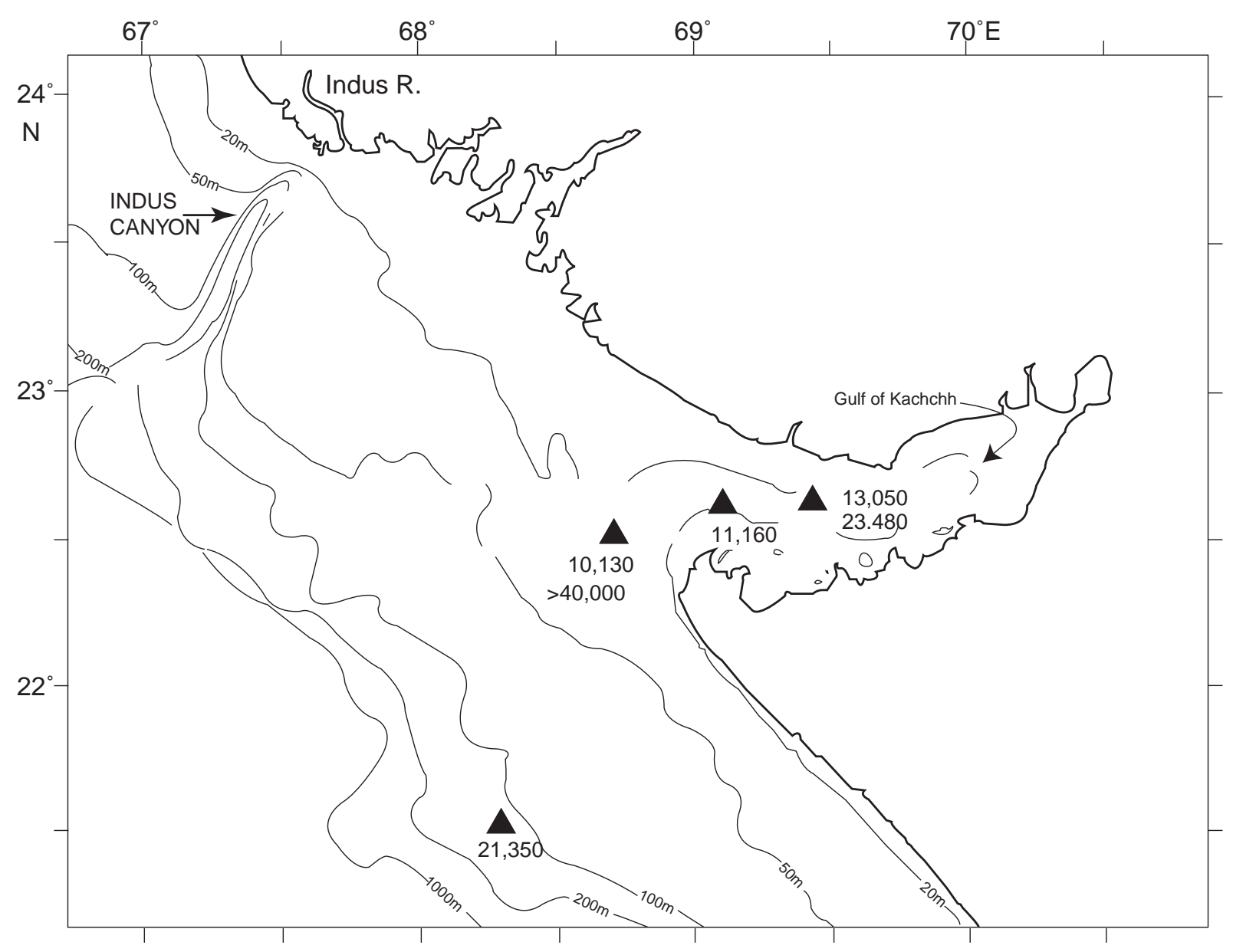

Figure 2(a). Measured radiocarbon ages of the samples off the Gulf of Kachchh.

consist of quartz, dolomite and high-magnesium calcite, whereas the associated sheet limestones consist of calcite and quartz. Thin sections indicate that the dolomite crusts consist of abundant thin dolomite micro-laminations or laminations with trapped sediment detritus (figure 3a-b). SEM studies indicate that these laminations are caused by encrustation of microbial filaments, which were dolomitized directly (figure 3c). Rod-shaped and elongated dolomite microparticles resembling fossilized bacteria are also abundant (figure 3d). Oxygen $\left(\delta^{18} \mathrm{O}=3.13 \%\right.$ o $)$ isotopes of the dolomite (table 1) indicate its formation in hyposaline conditions. The carbonate minerals (dolomite and highmagnesium calcite) forming the dolomite crust are marine. The ages of the dolomite crusts at $35 \mathrm{~m}$ and $25 \mathrm{~m}$ are $12,550{ }^{14} \mathrm{C}$ yr BP (14.3 ka) and 10,660 ${ }^{14} \mathrm{C}$ yr BP (12.5 ka), respectively. The corals at $25 \mathrm{~m}$ are dated at $10.8 \mathrm{ka}$. The ages of the sheet limestones $\left(22,980{ }^{14} \mathrm{C}\right.$ yr BP) and micritic limestones $\left(>40,000{ }^{14} \mathrm{C}\right.$ yr $\left.\mathrm{BP}\right)$ respectively are at the extreme end and beyond the ages datable by ${ }^{14} \mathrm{C}$ method (table 2; figure 2a).

\subsection{The carbonate platform}

The relic sediments on the carbonate platform are largely aragonite sands, which are tan in colour landward and shiny white offshore. Thin section studies on these grains indicate that some of these sands are Crustacean faecal pellet-dominated (Rao et al 1994), while others are oolite-dominated. Age of the aragonite sands ranges from 12,440 to 6,730 ${ }^{14} \mathrm{C}$ yr BP (14.3 to $\left.7.6 \mathrm{ka}\right)$. The sands at trough portions of sand ridges (see Wagle et al 1994) exhibit older ages than that of the crest (tables 1-2; figure 2b). Halimeda-dominated and pelletal/oolitic limestones and, indurated aragonite muds with several borings and serpulid encrustations, similar to hardground deposits, were found closer to the seaward edge of the platform (table 1). Acicular aragonites are the dominant cements in them. The ages of the limestones mostly lie between 9,920 and $11,480{ }^{14} \mathrm{C}$ yr BP (11 ka to $13.3 \mathrm{ka}$ ) (figure $2 \mathrm{~b}$ ). Relatively young corals $(2.7 \mathrm{ka})$ buried under siliciclastic sediments were recovered in one Peterson Grab station (SK-111/18), while at another 


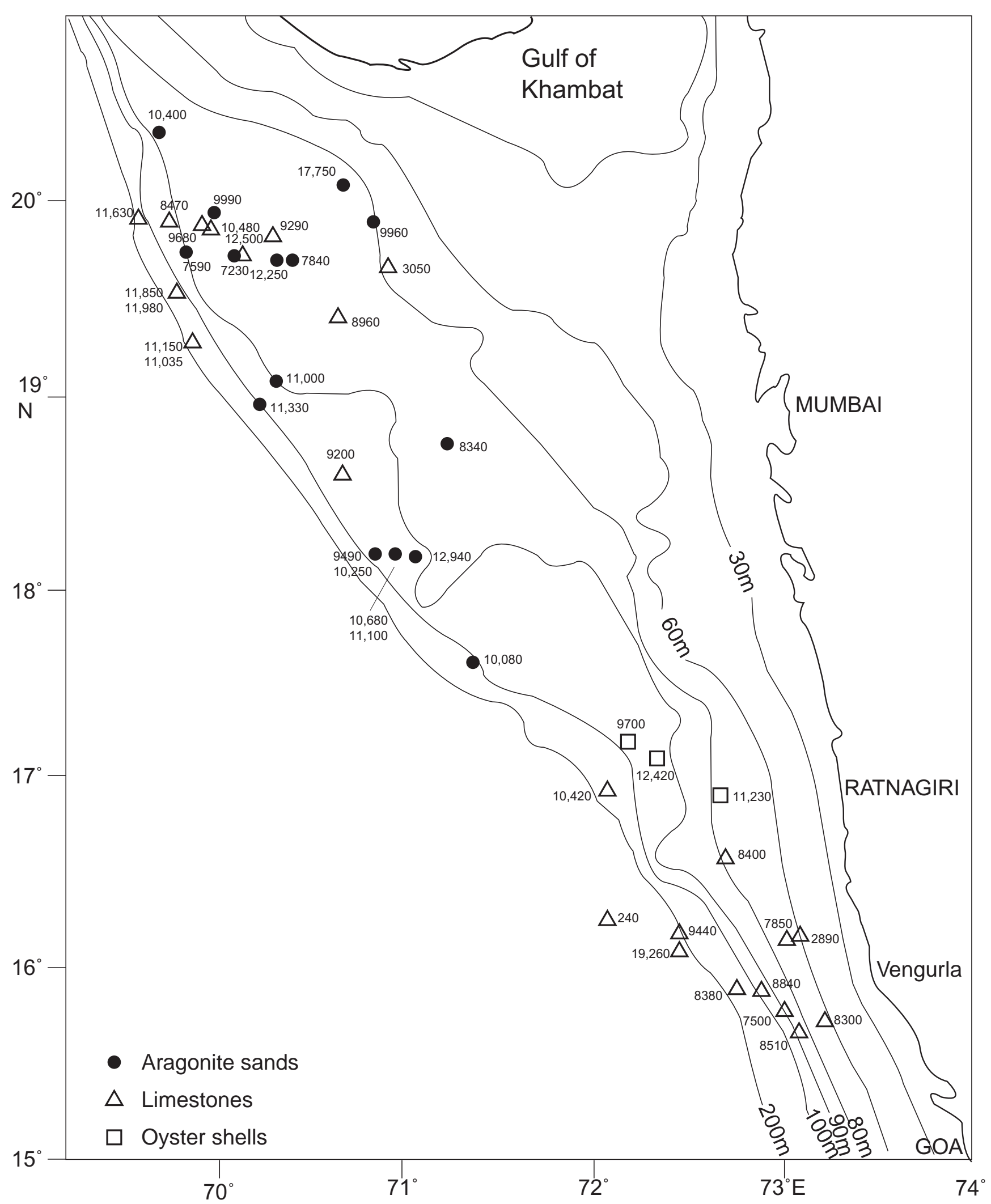

Figure 2(b). Measured radiocarbon ages of the samples on the carbonate platform and Ratnagiri-Goa. 


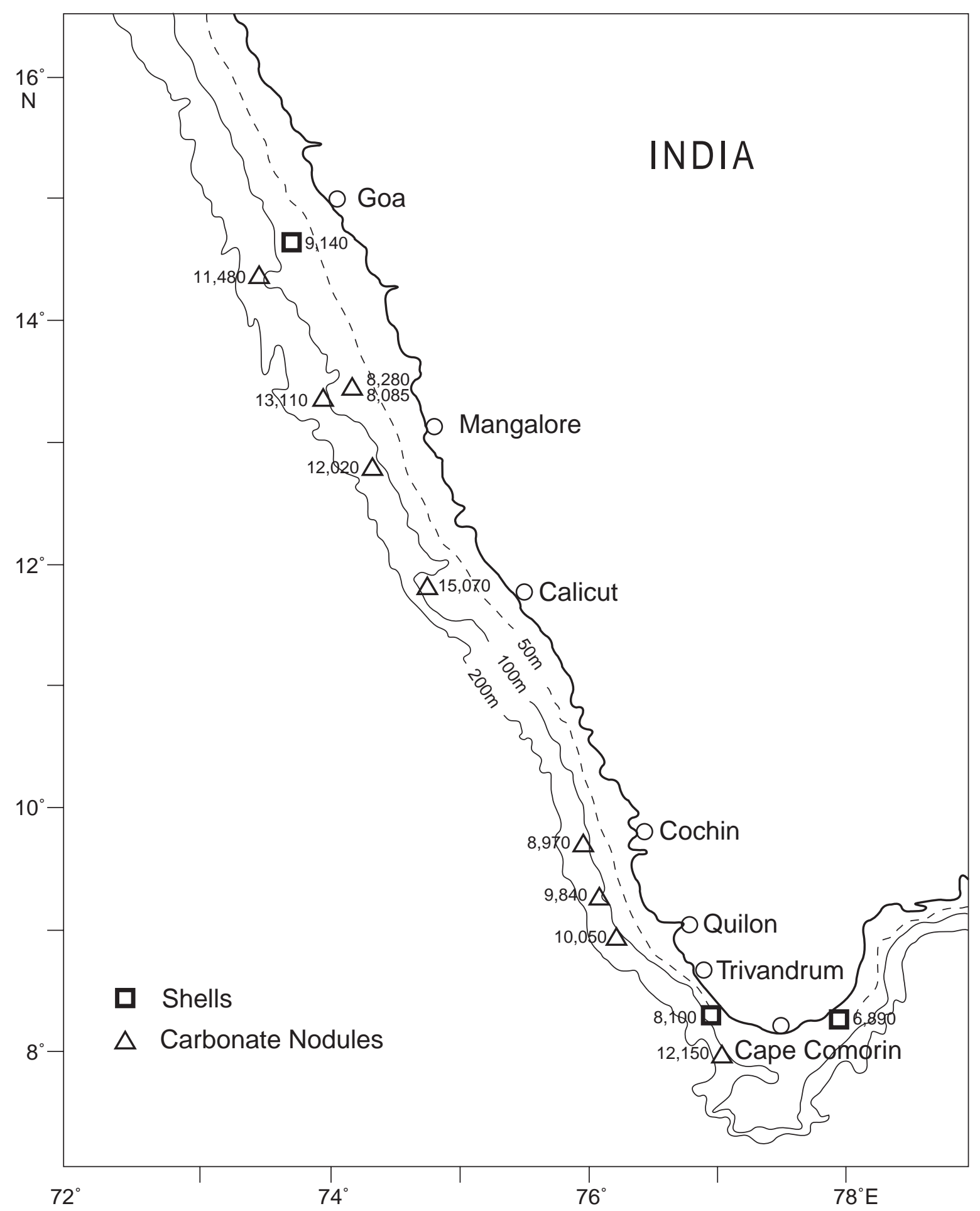

Figure 2(c). Measured radiocarbon ages of the samples off Goa-Cape Comorin.

station (SK-111/16) coralline-algal nodules (dated $12,000{ }^{14} \mathrm{C}$ yr BP) occur beneath the aragonite sands dated at $6,730{ }^{14} \mathrm{C}$ yr BP $(7.6 \mathrm{ka})$. A shell zone comprising of oyster shells of different sizes occurs off Ratnagiri (table 1; figure 2b; figure 4a). The largest shells have the oldest age $\left(11,920{ }^{14} \mathrm{C}\right.$ yr BP) (table 2). Dolomite crusts also occur at $64 \mathrm{~m}$ water depth on the northeastern edge of the platform. Dolomite, high-magnesium calcite and quartz are dominant minerals and, feldspar, marcasite and pyrrhotite are minor. SEM studies indicate that the crust contains irregularly laminated dolomitized cyanobacterial filaments (figure 5a) or laminated microbial mats with entrapped particles (figure $5 \mathrm{~b}-\mathrm{c}$ ) and dolomite microparticles $(5 \mu \mathrm{m})$ resembling fossil bacteria or their aggregates 

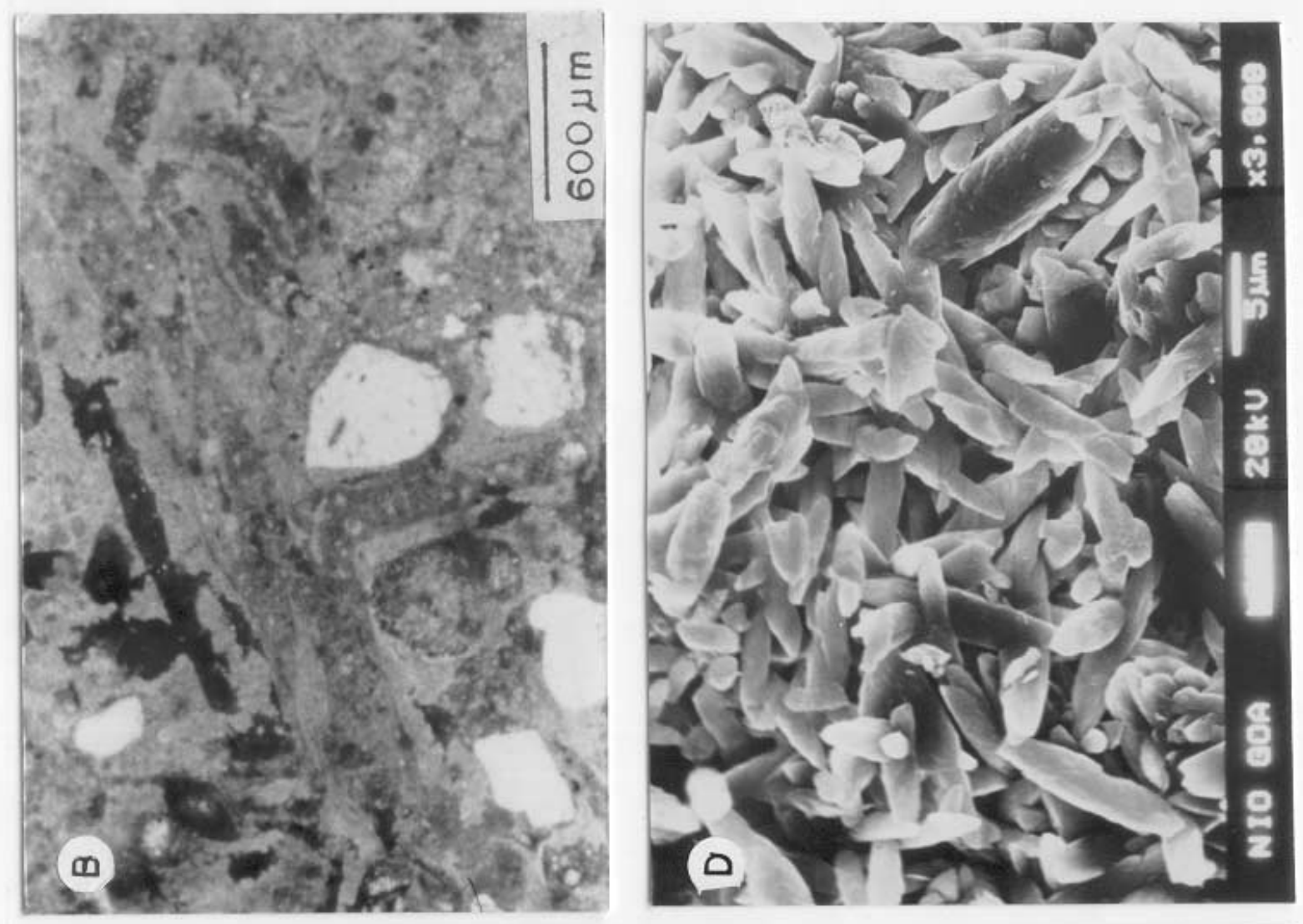

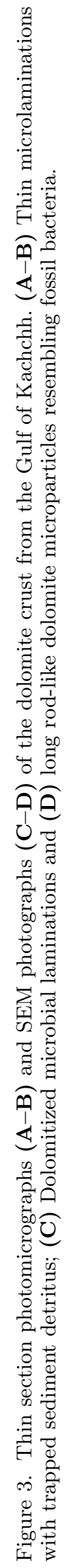
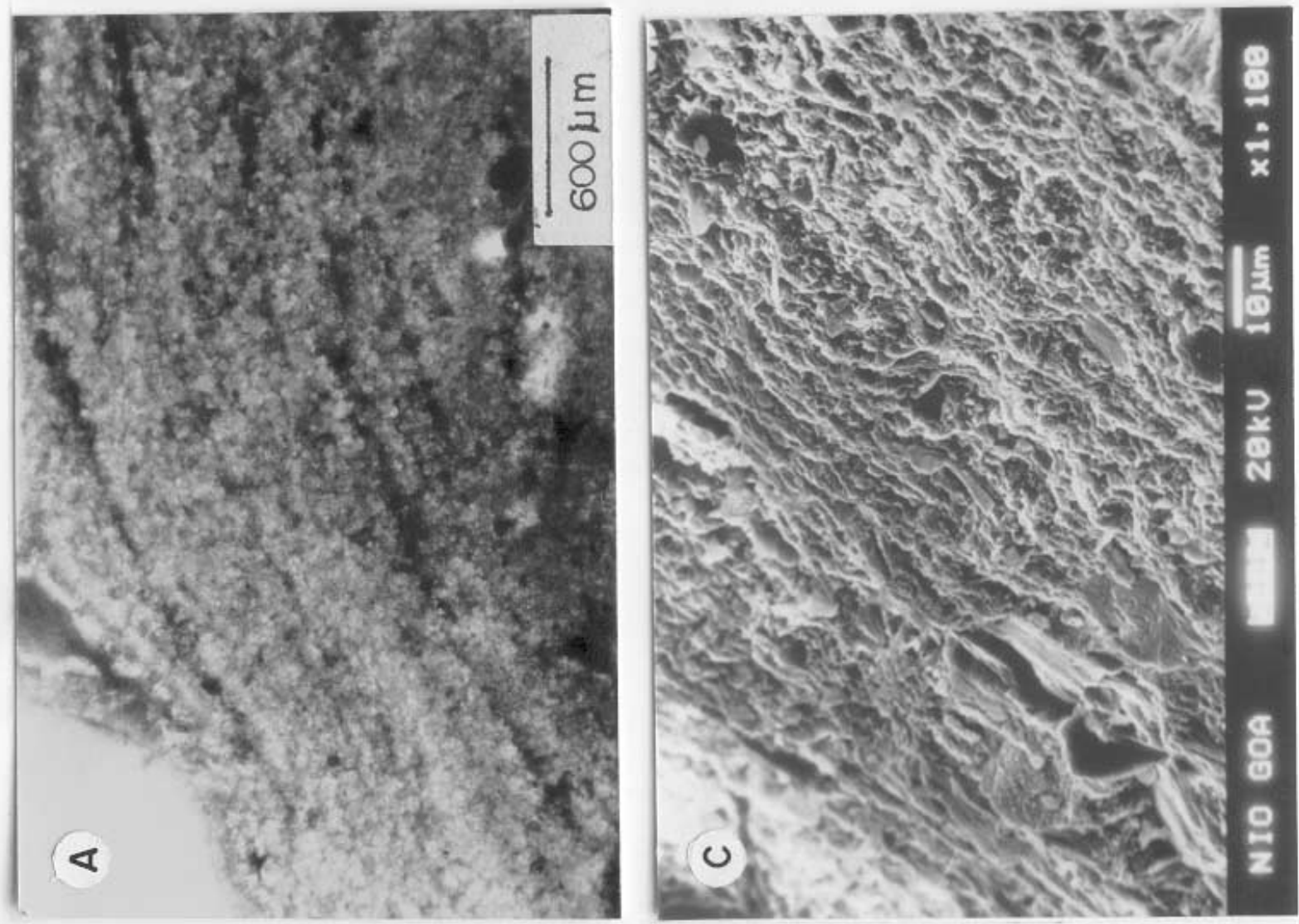

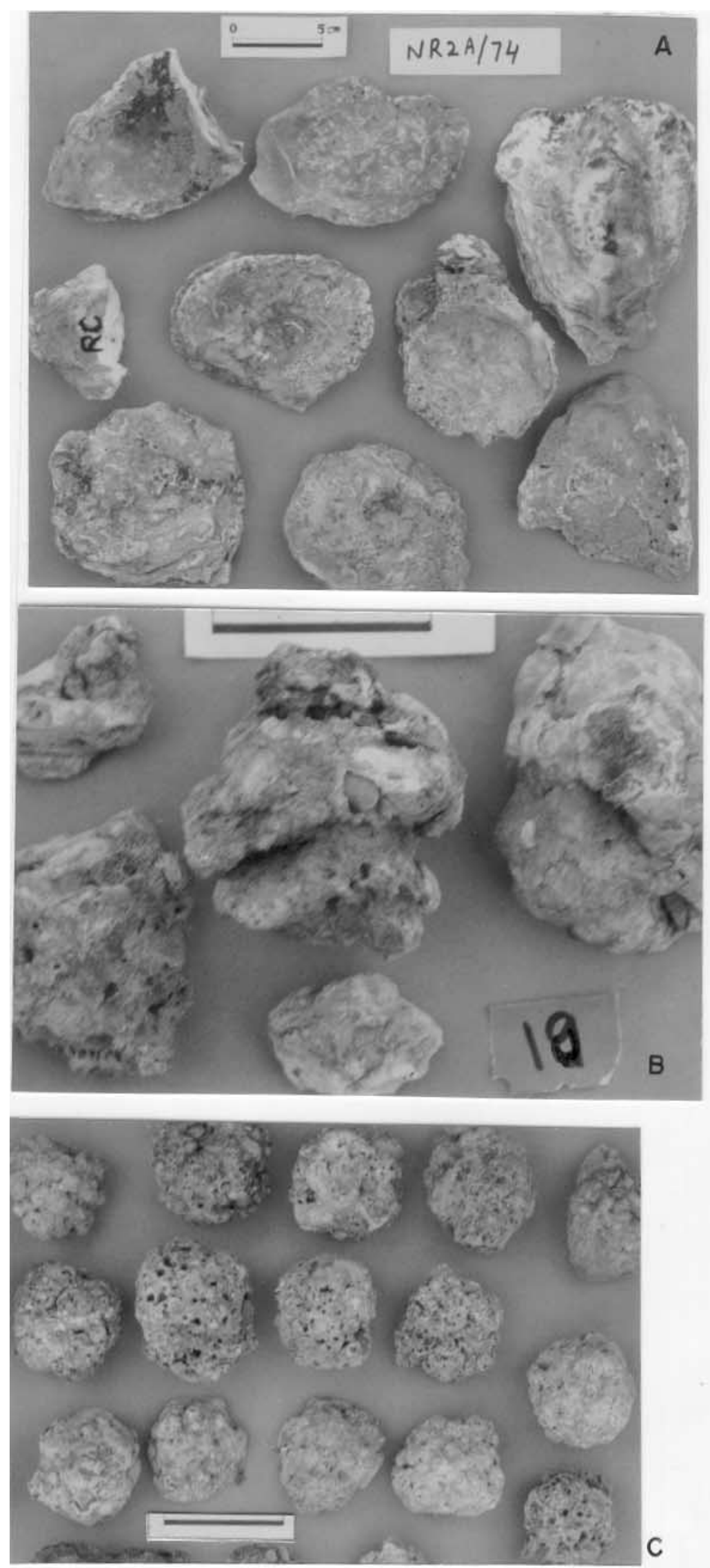

Figure 4. Hand specimens of samples: (A) Oyster shells from the carbonate platform, (B) Carbonate nodules off Vengurla and (C) off Cape Comorin. Scale bar is $5 \mathrm{~cm}$ long. 


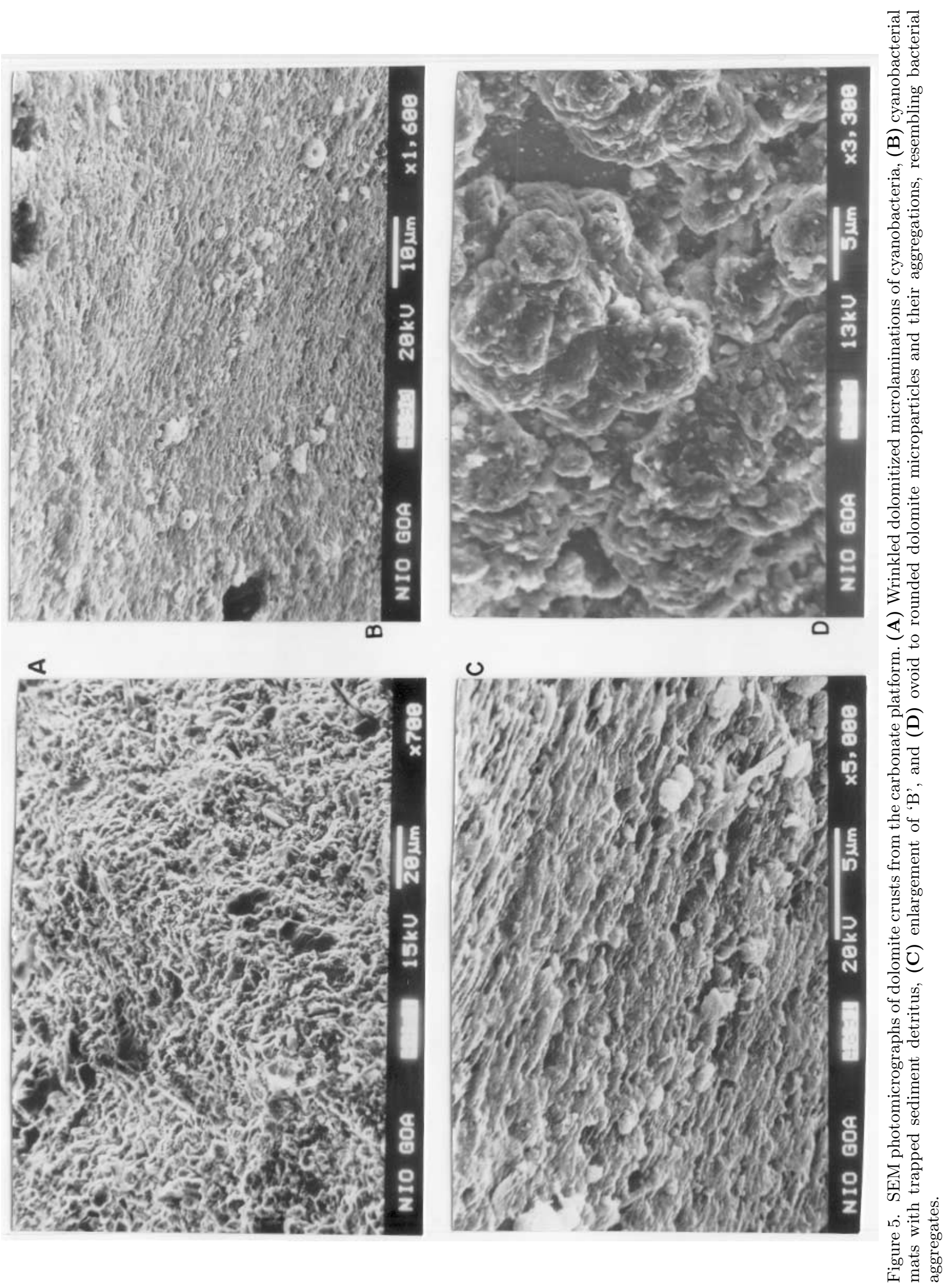


(figure $5 \mathrm{~d}$ ). Oxygen isotopes $\left(\delta^{18} \mathrm{O}=4.17 \%\right.$ o $)$ of the dolomite (table 1 ) indicate hypersaline conditions at the time of its formation. The AMS age of the crust is $17,250{ }^{14} \mathrm{C}$ yr BP (20.3 ka) (table 2; figure $2 \mathrm{~b}$ ). Dolomite crusts are the oldest samples so far reported for near surface sediments of the carbonate platform.

\subsection{Vengurla - Mangalore}

Grey carbonate nodules (figure 4b) were recovered abundantly between Vengurla and Goa and sparsely between Goa and Mangalore. Highmagnesium calcite, carbonate fluorapatite and quartz are present in order of abundance. These nodules contain up to $10 \% \quad \mathrm{P}_{2} \mathrm{O}_{5}$ (Rao and Lamboy 1996). Polished and thin sections indicate that the nodules are dominated by foraminiferal (Gypsina) encrustations (figure 6a) with intermittent thin coralline algal laminations (figure 6b-c). Mesophyllum (figure 6c), Lithothamnium, Archaeo-lithothamnium, Sporolithon, Lithoporella and Porolithon are the different genera of coralline alga present intermittently in different nodules. These associations represent moderate to deeper depths at the time of their formation (see Rao et al 2002a; Taberner and Bosence 1985; Adey 1986; Reid and Macintyre 1988; Minnery 1990). Siliciclastic detritus trapped between the laminations has been phosphatized. These nodules also show a succession of encrusters and are represented by alternate laminations of foraminifera and coralline algae, or repeated succession of foraminifera, coralline algae and encrusting corals (figure 6d). The age range of the nodules off Vengurla-Goa is narrow and lies between 7,000 ${ }^{14} \mathrm{C}$ yr BP $(7.9 \mathrm{ka})$ and $8,940{ }^{14} \mathrm{C}$ yr BP (10 ka), while that of Goa - Mangalore is slightly higher $\left(10,980{ }^{14} \mathrm{C}\right.$ yr BP) (12.9 ka) (table 2$)$. Corals overlain by encrusted foraminiferal laminae occur at $70 \mathrm{~m}$ water depth off Mangalore (SK65/2). The age of the coral is $7,780{ }^{14} \mathrm{C}$ yr $\mathrm{BP}$, while that of the foraminiferal encrustation is $7,590{ }^{14} \mathrm{C}$ yr BP. Coral polyps at $59 \mathrm{~m}$ depth are dated at $2,390{ }^{14} \mathrm{C}$ yr BP (2.5 ka) (table 2).

\subsection{Mangalore - Cape Comorin}

The ages of the Porites corals off Mangalore at 105 and $110 \mathrm{~m}$ water depths are $12,610^{14} \mathrm{C}$ yr BP and $11,520{ }^{14} \mathrm{C}$ yr BP, respectively (table 2 ). Spherical carbonate nodules $\sim 5 \mathrm{~cm}$ diameter (figure $4 \mathrm{c}$ ) occur abundantly at $90 \mathrm{~m}$ off Cape Comorin. Thin sections of the nodules indicate dense coralline algae (figure 7a) belonging to Lithothamnium and Lithophyllum with thin veneers of encrusting foraminifera. Solution features, alteration of skeletal material and micritisation of algal material (figure $7 \mathrm{~b}$ ) are common in these nodules. These features indicate shallow water conditions at the time of their formation. These nodules are dated at $11,650{ }^{14} \mathrm{C}$ yr BP (13.5 ka). Sandy sediments comprising of a mixture of benthic foraminifers and mollusks were recovered at the inner shelf around Cape Comorin. The age of these sandy sediments at $49 \mathrm{~m}$ is $7,600{ }^{14} \mathrm{C}$ yr BP $(8.4 \mathrm{ka})$ while those at $18 \mathrm{~m}$ depth is $6,390{ }^{14} \mathrm{C}$ yr BP (7.3 ka) (tables $1-2$; figure 2c).

\section{Discussion}

\subsection{Evidence of sea level during isotope stage 3}

The oldest samples recovered are sheet limestones from the Gulf of Kachchh. They are dominated by both low- and high-magnesium calcite. Their ages (table 2) provide evidence for sea level in the Gulf prior to the Last Glacial Maximum (LGM), i.e., in isotope stage 3 (50-26 ka), during which the global sea level was within -40 to $-80 \mathrm{~m}$ (Labeyrie et al 1987; Bard et al 1990).

\subsection{Sea level during LGM}

Dolomite crusts from the carbonate platform are the second oldest on this margin. The cyanobacterial mats, trapping and binding of sediments by microbial mats and their direct dolomitization (figure $5 \mathrm{a}-\mathrm{d}$ ) indicate that these dolomite crusts were similar to dolomite stromatolites. The origin and environmental conditions of the crusts in detail were discussed elsewhere (Rao et al 2002b), suggesting that the dolomite formed in the crusts is primary, microbially mediated and in shallow (subtidal to intertidal) hypersaline conditions. The other carbonate phase - high-magnesium calcite associated with the crust is also of marine origin. The AMS age of the crusts at $64 \mathrm{~m}$ is $17,250 \mathrm{yr}$ BP. Contrastingly, the glacio-eustatic sea level at about 18,000 yr BP was at $-120 \mathrm{~m}$ (Fairbanks 1989). This implies, unlike other continental shelves of the world oceans or continental shelf between Mangalore-Cape Comorin (see below) which were subaerially exposed during the last glacial maximum (LGM - 18,000 yr BP), the carbonate platform was at shallow depths or pockets of lagoons may have existed on the platform in which the dolomite crusts were formed. Hypersaline conditions revealed by stable isotopes of the crusts (table 1) point to the existence of lagoons on the carbonate platform. Since these crusts were collected by Peterson Grab, the lagoons may not have been too deep. In other words, the platform was 

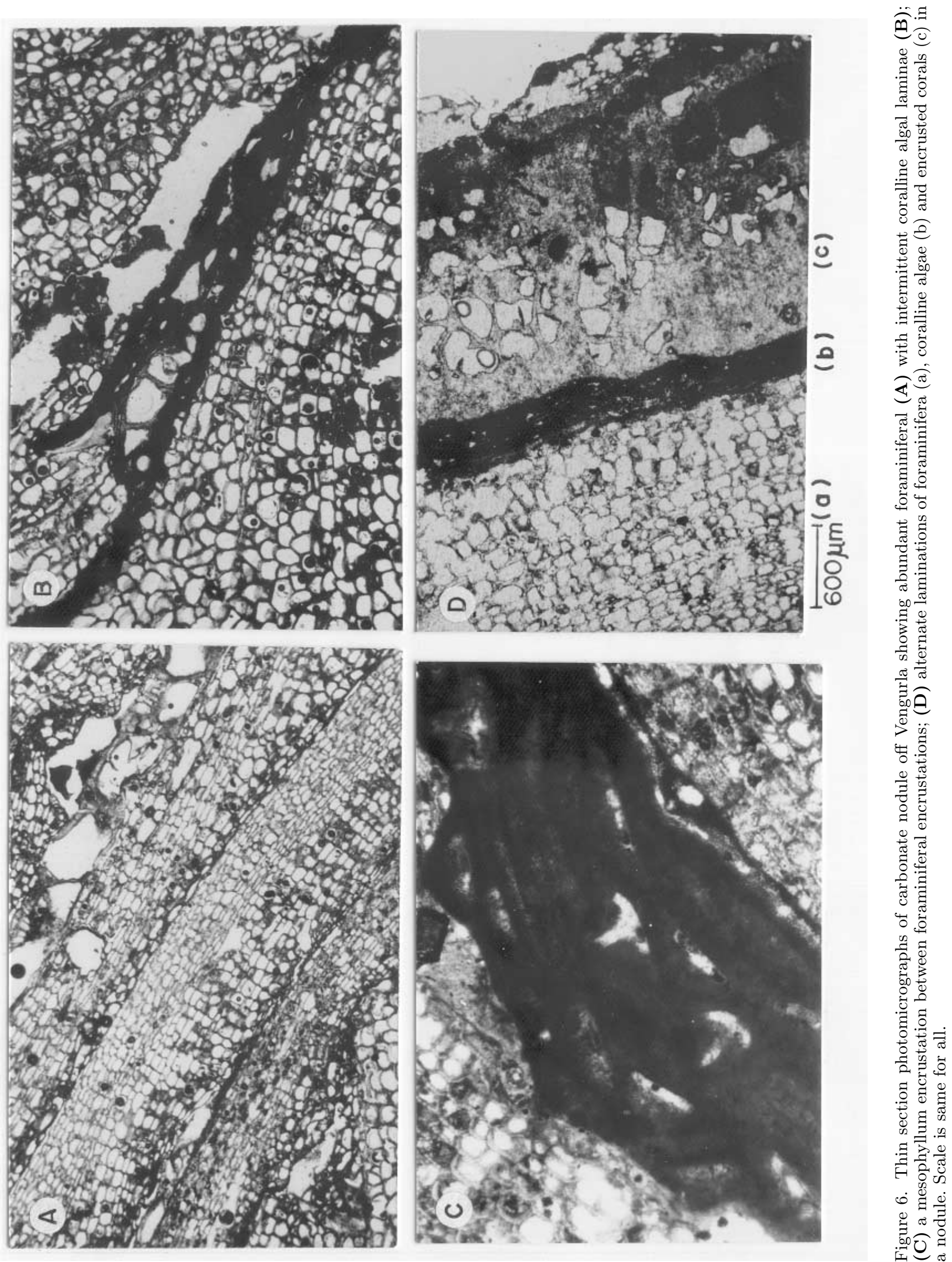


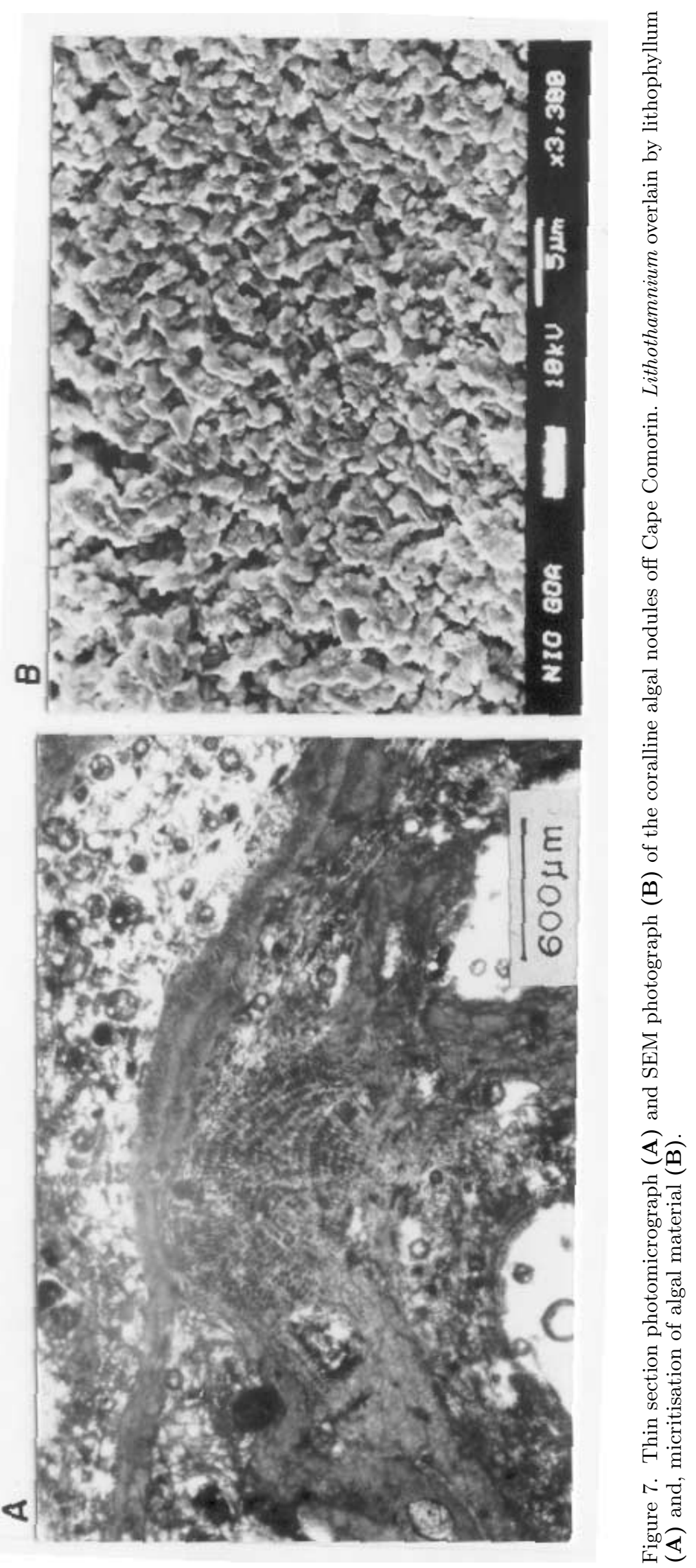


at shallow depths during the LGM and explanation is needed for the difference of sea level of $\sim 50 \mathrm{~m}$ at about LGM between the global position $(-120 \mathrm{~m})$ and the platform position $(-64 \mathrm{~m})$. The next higher or older age $\left(12,440{ }^{14} \mathrm{C}\right.$ yr $\left.\mathrm{BP}\right)$ comes from the aragonite sands of the platform. Since both (dolomite crust and aragonite sands) are near surface sediments and are separated by $\sim 5,000$ years in age, we presume that the carbonate production was low on the platform between 17,250 and $12,440{ }^{14} \mathrm{C}$ yr BP. Beck et al (2001) reported decreased atmospheric $\Delta^{14} \mathrm{C}$ values from $\sim 700$ to $\sim 100$ per mil between 26 and $11 \mathrm{ka}$ and suggested substantially slower or shallower thermohaline circulation and/or reduced carbonate sedimentation rates are necessary to explain these values.

\subsection{Sea level during 12,610 - 11,650 yr BP}

The sediments (or sedimentary rocks) with radiocarbon ages between $12,610{ }^{14} \mathrm{C}$ yr BP (14.8 ka) and $11,650{ }^{14} \mathrm{C}$ yr BP $(13.5 \mathrm{ka})$ were spread along the margin in the form of different deposits (figure 2a-2c; table 2). They occur as dolomite crusts in the Gulf of Kachchh, coralline algal nodules, aragonite sands or oyster shells (figure 4a) on the carbonate platform, Porites corals off Mangalore and coralline algae-dominated nodules (figure 4c) off Cape Comorin (tables 1 and 2). These deposits, however, occur in the depth range of 85 - $110 \mathrm{~m}$ between Cape Comorin and Ratnagiri and on carbonate platform, but at $35 \mathrm{~m}$ in the Gulf of Kachchh (table 1). Interestingly, some of these are shallow water deposits and serve as sea level indicators. For example, live Porites corals with a cap of $<10 \mathrm{~m}$ water are found abundantly in the Indo-Pacific region (Done 1982; Veron 1986). Dense coralline algae and its alteration to micrite (figure 7a-b) in the nodules off Cape Comorin are indicative of shallow marine conditions at the time of their formation (see Logan et al 1969; Winland 1969; Bosence 1985; Rao et al 2002a). Similarly, oyster shells (figure 4a) were reported at intertidal to subtidal depths and have been used as sea level indicators (Merrill et al 1965; Meldhal and Cutler 1992). These imply that the sea level reached about $85 \mathrm{~m}$ by about $13.5 \mathrm{ka}$ along the western margin of India between Cape Comorin and Mangalore. Since dolomite crusts dated 17,250 yr BP and different deposits (oyster shells, aragonite sands and algal nodules occupying different latitudes) having age $\sim 12,000$ yr BP (table 2; figure 2c) are present on the platform, it is difficult to envisage the water depth on the platform during this time. Although the depth of the shell zone off Ratnagiri (southern part of the platform) coincides very well with glacio-eustatic sea level position, the depth and age of the aragonite sands do not correspond (see below). At present we are unable to answer this discrepancy. Relic reefs at about 100 to $90 \mathrm{~m}$ containing sediments/reefs of $\sim 12,000{ }^{14} \mathrm{C}$ yr BP were reported off Caribbean Islands, Comoro Islands and Red Sea (Dullo et al 1998; Montaggioni 2000). Our findings for this period of time between Cape Comorin and Ratnagiri are consistent with other margins.

The depth and ages of the samples from the Gulf of Kachchh are in contrast with that on the glacio-eustatic sea level. Dolomite crusts dated at $14.3 \mathrm{ka}$ occur at $35 \mathrm{~m}$ in the Gulf. Dolomite usually forms under evaporative conditions and at shallower depths on the continental shelf, where large volumes of normal sea water/brines are pumped through the sediments (Patterson and Kinsman 1982; Carballo et al 1987). Moreover, laminated microbial mats with intermittent sediment particles (figure $3 \mathrm{a}-\mathrm{d}$ ) are considered to form at shallow depths (Logan et al 1969; Walter 1976). Dolomite encrustations occur on large sheet limestones and are formed by dolomitization of microbial filaments. We, therefore, consider that these crusts were developed in situ. Rugged topography with pinnacles as high as $10 \mathrm{~m}$ is characteristic of the sea floor at the mouth of the Gulf of Kachchh ruling out the possibility of transportation. Since miliolite limestones are spread along the Saurashtra, one would argue that the detrital carbonates from these limestones may have transported and mixed up with these dolomites and diluted the age of the dolomite crust. The dolomite crusts consist of abundant dolomites of primary origin formed by microbial mediation (figure $3 \mathrm{a}-\mathrm{d}$ ) and highmagnesium calcite, both formed in hyposaline conditions (see stable isotopes). Since low-magnesium calcite (major mineral of the miliolite limestones on land) is not present in the crusts, contamination from older carbonate detritus may not be a major factor. Furthermore, the ages of Favia corals (aragonite in composition) (10.8 ka) and dolomite crust $(12.5 \mathrm{ka})$ at 28 and $25 \mathrm{~m}$, respectively at the mouth of the Gulf (see tables 1 and 2) also do not correspond with the glacio-eustatic sea level (see below). Aragonite crust dated at $13.7 \mathrm{ka}$ occurs in a core (at 100-105 cm interval) collected at $55 \mathrm{~m}$ depth on the shelf off the Gulf of Kachchh (V P Rao unpublished data). These suggest that not only dolomite crusts but also other types of samples (aragonite crusts and corals) collected from the Gulf and in its vicinity showed similar older ages. We, therefore, consider that the ages of the crusts are reliable. Since the oldest age of the sample (dolomite crust) is 14.3 ka the Gulf must have inundated much before, because for initiation of dolomite formation (or for the growth of corals) one would expect a lag period after flooding of the Gulf. Davies and Montaggioni (1985) reported a lag period of $1200-2000$ years 
between initial flooding and start-up phase for the coral settlement in the Great Barrier Reef (GBR). Keeping this in view, we suggest that after the Last Glacial Maxima (LGM), the Gulf must have inundated atleast by $\sim 15$ ka. Corals of the Faviidae family are characteristic of turbid water and occur at shallow depths (1-6 m) (Veron 1986). These corals occur at $28 \mathrm{~m}$ in the Gulf and are dated at $10.8 \mathrm{ka}$. The glacio-eustatic sea level was, however, at $\sim 70 \mathrm{~m}$ by $11 \mathrm{ka}$ (Fairbanks 1989). This implies that the Gulf of Kachchh was uplifted atleast by $\sim 40 \mathrm{~m}$ sometime after $10.8 \mathrm{ka}$.

The Kachchh region is seismically active and controlled by a series of normal and low-angle reverse faults, which are exposed in certain regions. Several $\mathrm{Th}^{230} / \mathrm{U}^{234}$ ages on miliolite limestones of Saurashtra and Kachchh indicated three episodes of uplift and miliolite deposition during the Pleistocene (Baskaran et al 1989). Recent regional studies have shown the uplift of the northern Kachchh at different times in Holocene, including 8,500 yr BP, 5,000 yr BP and 2,500 yr BP (Parkash et al 2000) and subsidence of Bet Dwarka sometime after 3,870 yr BP (Rao 1996; Vora et al 2002). Although the uplift time at 8,500 yr BP (9.6 ka) on land is close to the younger age of the sample (coral - 10.8 ka) from the Gulf of Kachchh, other regional uplift times and subsidence time of the Bet Dwaraka cannot be accounted with our data. Palaeoseismological studies further indicate that the Kachchh region experienced large and moderate earth quakes, including the ones in the most recent years $(1891,1956$ and 2001). Some of these earthquakes are caused by blind faults and induced surface deformation and widespread liquefaction including lateral spreading (see Rajendran et al 2001). We, therefore, suggest that the Gulf of Kachchh was subjected to complex tectonic movements (including uplift and subsidence at different times) during the late Pleistocene and Holocene and it is difficult to quantify the rate of uplift/subsidence.

\subsection{Sea level between 10,000 and 7,000 yr BP}

The age of the aragonite sands (oolites and aragontic faecals) from 15 stations on the carbonate platform (figure $2 \mathrm{~b}$ ) ranges between 12,440 and 6,730 yr BP implying that carbonate sedimentation continued until 6,730 yr BP. The colour differences of these sands from east to west of the platform suggest that there is no mixing of sands from different depths. If one considers that oolites form in shallow marine conditions and serve as sea level indicators (Illings 1954; Loreau and Purser 1993), it is necessary to envisage intertidal to subtidal conditions on the platform during their formation. This seems unlikely here, because the glacio-eustatic sea level rose by $\sim 80 \mathrm{~m}$ between 12,000 and $7,000 \mathrm{yr} \mathrm{BP}$. This implies that oolites may not be considered as sea level indicators and can even form at deeper depths (Kump and Hine 1986; Rao et al 1996). Rao et al (1994) suggested that some of these sands are aragonitic faecals and, Halimeda bioherms acted as the source of aragonite mud for the formation of faecals. It is also difficult to explain the continued growth of Halimeda bioherms with the rise of sea level by about $80 \mathrm{~m}$.

The limestones (G72/1459, G72/1479) collected at $95 \mathrm{~m}$ and $85 \mathrm{~m}$ water depth on the platform show solution features, needle fibre aragonite and drusy calcite cements indicating vadose diagenetic conditions of the platform. The age of the limestone at $85 \mathrm{~m}$ depth is about $8,785{ }^{14} \mathrm{C}$ yr BP (Rao et al 1994) (table 2; figure 2b). The glacio-eustatic sea level at 9,000 yr BP was $\sim-37 \mathrm{~m}$ (Fairbanks 1989) implying that a difference of sea level of about $\sim 50 \mathrm{~m}$ have to be explained.

\subsection{Age vs depth relation of the samples}

Forty-six of the 62 data points from table 2 were plotted on the glacio-eustatic sea level curve of Fairbanks (1989) (see figure 8). The age/depth of the samples which are outside the limits of the curve, duplicate analyses, age of the samples from the gravity cores, age of the corals from the Angria Bank and ages of the polyp corals (see table 1) were not plotted on this curve. The samples from the Gulf of Kachchh fall distinctly on the right of the curve (figure 8). As explained earlier, this is due to early flooding of the Gulf atleast by $15 \mathrm{ka}$ after the Last Glacial Maxima and subsequent uplift and/or subsidence. The dolomite crusts from the platform also fall on the right of the curve. All other samples plot on or left of the curve. There are a few data points at depths $<60 \mathrm{~m}$. Only 8 samples were dated from the continental shelf between Cape Comorin and Mangalore. Of these, five samples at water depths from 110, 90, 58, 49 and $18 \mathrm{~m}$ having their ages between 12,610 and $6,390 \mathrm{yr} \mathrm{BP}$ fall on or, very close to the glacio-eustatic sea level curve. On the other hand, the ages of the oyster shells and a few coralline-algal limestones from the platform also fall very close to the sea level curve, but all other samples from the platform fall away from the curve (figure 8). In other words, the samples dated 12,000 - 6,900 yr BP from the carbonate platform spread at depths between 105 and $65 \mathrm{~m}$, whereas the samples of the same age range from the shelf between Cape Comorin and Mangalore occur at depths between 110 and $18 \mathrm{~m}$. This suggests that carbonate sedimentation continued on the platform despite the glacio-eustatic sea level rise by about $80 \mathrm{~m}$ (between 12,000 and $7,000 \mathrm{yr} \mathrm{BP}$ ) or, the platform was subjected to 


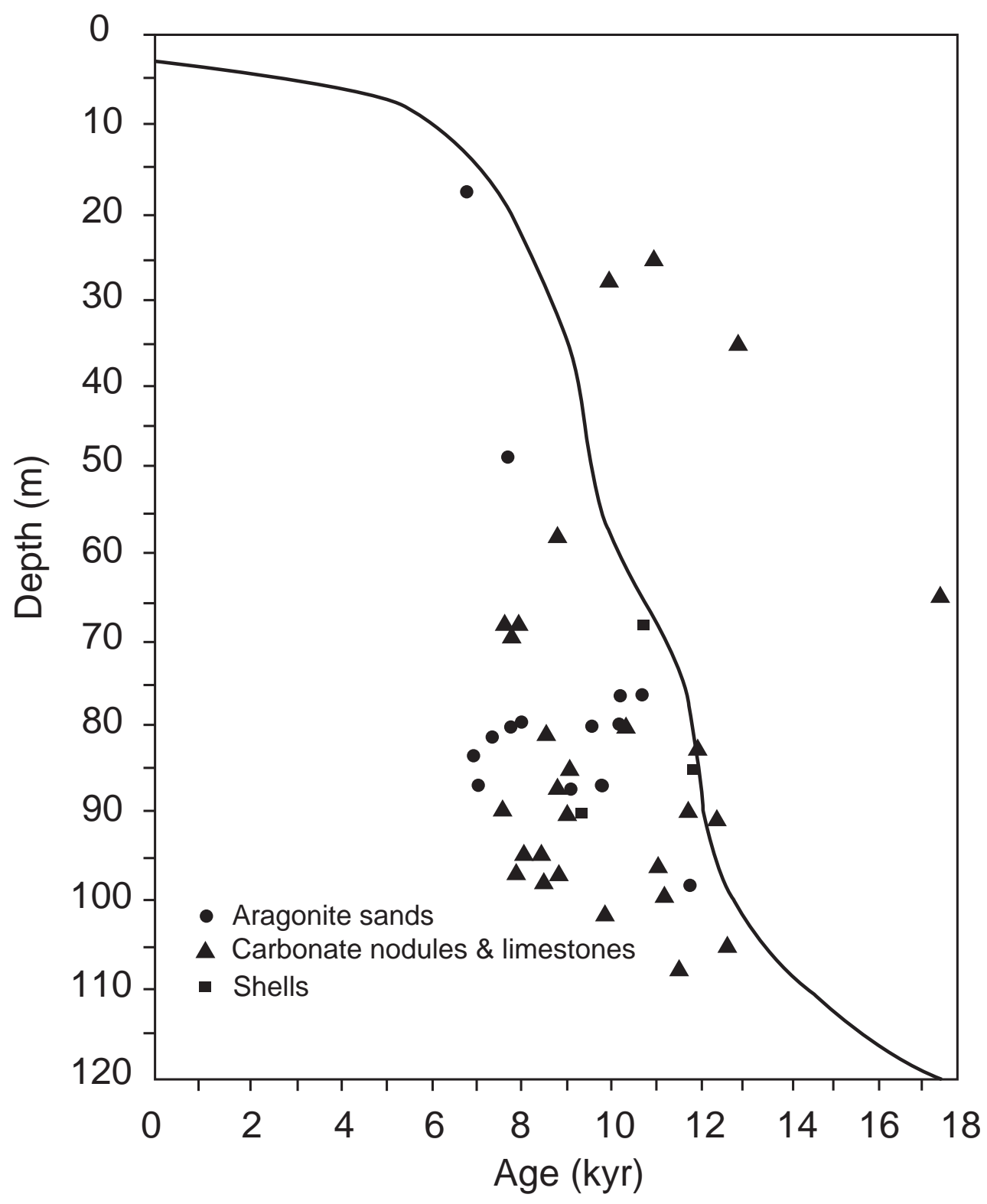

Figure 8. A plot of corrected radiocarbon ages and depth of the samples on a glacio-eustatic sea level curve of Fairbanks (1989).

intermittent neotectonic activity and then carbonate growth continued. We are indeed concerned and careful about documenting the neotectonic activity on the platform, as many do not believe this possibility on the passive margins such as Indian margins. However, evidences such as (a) deeper water terraces (Rao and Veerayya 1996), (b) limestones of early Holocene age showing vadose diagenetic textures (see Rao et al 1994), (c) dolomite crusts dated $17,250 \mathrm{yr} \mathrm{BP}$ at $64 \mathrm{~m}$ depth and (d) age of the aragonite sands $(12,440-6,730 \mathrm{yr} \mathrm{BP})$ on the platform cannot be explained by any other single mechanism. Even considering that aragonite on the platform is of biogenic origin (derived from $\mathrm{Hal}$ imeda bioherms), it is difficult to envisage a mas- sive carbonate production with the rise of sea level about $90 \mathrm{~m}$, between $12,440 \mathrm{yr}$ and the present. It is known that the glacio-eustatic sea level reached its present position at about 6000 years BP. If one assumes aragonite forms even in deeper water, the controlling factor for the cessation of aragonite sands after 6,730 years BP, and absence of terrigenous sediments, between 12,440 yr BP and the present, on the platform despite its position off the rivers need explanation. Keeping these in view, we believe that the platform may have been at a different elevation during LGM and subsequently subsided during the Holocene. The exact time/times and rate/rates of subsidence are unknown and it is difficult to quantify the phases of subsidence with 
the existing data. Since aragonite cemented vadose diagenetic limestones (G72/1459) were formed at $8.85 \mathrm{ka}$, we presume that atleast one phase of subsidence of the platform must be after $8.85 \mathrm{ka}$. The carbonate platform was an isolated feature during the Eocene. The sediments originated from the River Indus accumulate abundantly on the western slopes of the platform and sediments from the Narmada and Tapti on the eastern slope of the platform and in the Dahanu depression (inner shelf) since Eocene. The sediments thus accumulated on the slopes of the platform acted as load on the platform. The combined effect of water and sediment load may have been responsible for the subsidence of the platform.

\subsection{Inferred environmental conditions}

The relic ages of the Porites reefs (14.8 to $13.4 \mathrm{ka}$ ) off Mangalore indicate reef development stopped after $13.4 \mathrm{ka}$. There may be two reasons for their demise. The reefs may have been affected by the rapid changes in sea level caused by the main phase of deglaciation and to the melt water pulse (MWP) 1 A (Fairbanks 1989) or catastrophic rise events (Blanchon and Shaw 1995) occurred at about $14 \mathrm{ka}$. Palaeoclimate records from several sites of the western margin of India suggest enhancement of monsoonal precipitation about $15 \mathrm{ka}$ (Thamban et al 2001) and intensified precipitation between 11 and $9 \mathrm{ka}$ (Van Campo 1986). Such intensification could have resulted in large flux of fresh water and sediment into coastal water thereby reducing water transparency, salinity and increasing nutrient flux and creating eutrophic conditions. Such conditions are considered to inhibit coral growth (Hallock and Schlager 1986). The rapid sea level rise also interrupts sedimentation and starving of sediments, that would correspond to diastem recording a typical drowning unconformity. Hardground conditions are indeed evident at about this time (13.1-12.3 ka) on the relict deposits occurring at the seaward edge of the carbonate platform and are represented as indurated aragonite muds, serpulid encrustations on algal nodules or Halimeda or pelletal/oolitic limestones with several borings (tables 1 and 2; figure $2 \mathrm{~b})$.

Patches of coral fragments dated $7,780{ }^{14} \mathrm{C}$ yr $\mathrm{BP}$ and $2,550{ }^{14} \mathrm{C}$ yr $\mathrm{BP}$ or polyp corals dated $2,390{ }^{14} \mathrm{C}$ yr BP occur at depths between 60 and $70 \mathrm{~m}$ off Mangalore, carbonate platform and Vengurla (tables 1 and 2). These indicate intermittent oligotrophic conditions (atleast locally) on the shelf during which corals were formed. Present day corals (dated $240 \mathrm{yr} \mathrm{BP}$ ) at $22 \mathrm{~m}$ on the Angria Bank (SK111/1), located away from the continental shelf, indicate suitable conditions for reef development in the shallow offshore banks.
It is indeed amazing how carbonates developed when one considers the geographic settings of the platform, age of the sediments on it and environmental conditions during the early Holocene. Firstly, the platform is located off the river mouths of Narmada and Tapti, which debouch 58.7 million cu. m. of water and several tons of suspended and bed load annually through the Gulf of Khambat (Rao 1975). The water depths on the platform range between 60 and $110 \mathrm{~m}$. Since the glacioeustatic sea level at 10,000 yr BP was at $60 \mathrm{~m}$ (Fairbanks 1989), one would expect the platform at or slightly below the sea level at this time and river discharged sediments transported right onto the platform. Secondly, enhanced monsoonal conditions have been reported during 13,000 - 6,000 yr BP (Van Campo 1986; Sarkar et al 2000; Thamban et al 2001). As a consequence, one would expect large fresh water and siliciclastic flux during this time. Thirdly, it is well known that carbonate sedimentation rates are inversely proportional to the siliciclastic flux. In view of the above, one would expect abundant terrigenous flux and, as a result cessation of carbonate growth on the platform. However, carbonate sedimentation continued until 6,730 yr BP and the platform sediments contain only $<10 \%$ terrigenous flux. This implies the riverine flux delivered either filled the innershelf Dahanu Depression - wherein sediments are being accumulated since Eocene, and/or diverted southwards under the influence of southwest monsoon current (Banse 1968). If the latter is the case, one would expect a high degree of turbidity in the water column on the shelf south of the carbonate platform. The age of the foraminiferal-dominated nodules (figure 4b) (9,400 and 7,500 yr BP) off Vengurla - Goa (figure 2b) (south of the platform) indicates their formation during intensified monsoonal conditions. The constituents of the nodules indicate their deeper water origin. We therefore propose that these nodules are characteristic of turbid waters and support our argument that the riverine flux was directed southwards for the reasons given below.

- Hottinger (1983) and Plaziat and Perrin (1992) reported well-developed abundant foraminiferaldominated nodules and indicated that they even survive in turbid conditions, unfavourable for encrusting corallines.

- The intermittent micro-laminations of coralline algae probably represent seasonal growth (figure $6 \mathrm{~b}-\mathrm{c}$ ) when waters were more transparent under non-monsoonal seasons. During monsoonal months foraminifera are favoured due to excess turbidity which excludes coralline algae (see Reid and Macintyre 1988).

- Phosphatized sediment detritus trapped between laminations indicate abundant flux to the region 
and suboxic conditions at or closer to the sea bed at the time of their formation. Intensified monsoons and associated upwelling brings large nutrient input and reduces the temperature and dissolved oxygen contents at subsurface depths and these conditions are not conducive for corals and coralline algal growth. The cyclic bands of foraminifera, coralline algae and corals (figure $6 \mathrm{~d}$ ) in the nodules may indicate improvement in environmental conditions in the water column from turbid to eutrophic to clearer and oligotrophic at the time of their formation.

\section{Conclusions}

- Relic carbonate deposits on the western margin of India occur as dolomite crusts, aragonite sands, vadose diagenetic limestones, coralline algal and foraminiferal-dominated nodules, oyster shells, Faviidae/Porites corals and benthic foraminifera/mollusk-dominated sediments.

- The relic deposits dated $\sim 12,000-6,390{ }^{14} \mathrm{C}$ yr BP spread on the outer and inner continental shelf at depths of $110-18 \mathrm{~m}$ between Cape Comorin and Mangalore, but occur within a narrow depth range $(105-65 \mathrm{~m})$ on the carbonate platform of the northwestern margin of India. Relic deposits of the Gulf of Kachchh (25 - 35 m) are dated at $12,550-9,630{ }^{14} \mathrm{C}$ yr BP. Relic deposits of the isotope stage 3 also found in the Gulf.

- The age vs depth plot of the samples indicates that the Gulf of Kachchh was uplifted and the carbonate platform subsided during early Holocene. A few samples were dated on the shelf off Cape Comorin-Mangalore. Of these five samples plot on or, close to the glacio-eustatic sea level.

- Occurrence of Porites corals at 12,610 to 11,520, $\sim 7,780$ and $\sim 2,550{ }^{14} \mathrm{C}$ yr BP indicate intermittent oligotropic conditions. Ecological succession from corals to coralline algae and to foraminiferal encrustated nodules, indicates changing water column conditions with respect to the nutrients and turbidity, probably due to intensified monsoons during the early Holocene.

- The sediments of the Narmada and Tapti were largely deposited on the inner shelf or, diverted southwards during the early Holocene, facilitating carbonate growth on the platform and foraminiferal nodules off Vengurla-Goa.

\section{Acknowledgements}

We thank Dr. E Desa, Director of the National Institute of Oceanography, Goa for the encour- agement. We thank the Editor, Earth and Planetary Sciences and anonymous reviewers for constructive comments on the earlier manuscript. This work was financed by the Research Project (No. ESS/CA/A1-25-97) 'Sea level changes along the western margin of India during the Late Quaternary' of the Department of Science and Technology, New Delhi. This work is NIO contribution no. 3805.

\section{References}

Adey W H 1986 Coralline algae as indicators of sea level; In: Sea level Research - A manual for the collection and evaluation of data (ed) O Van de Plasche (Free University, Amsterdam, Geobooks, Norwich) p. 229-280

Banse K 1968 Hydrography of the Arabian Sea shelf of India and Pakistan and effects on temporal fishes; Deep Sea Research 15 45-79

Bard E, Hamelin B and Fairbanks R G 1990 U/Th ages obtained by mass spectrometry in corals from Barbados: sea level during the past 130,000 years; Nature 345 405408

Baskaran M, Rajagopalan G and Somayajulu B L K 1989 ${ }^{230} \mathrm{Th} /{ }^{234} \mathrm{U}$ dating of Quaternary carbonate deposits of Saurashtra, India; Chemical Geology 79 65-82

Beck J W, Richards D A, Edwards R L, Silverman B W, Smart P L, Donahue D J, Hererra-Osterheld S, Burr G S, Calsoyas L, Jull A J T and Biddulph D 2001 Extremely large variations of atmospheric ${ }^{14} \mathrm{C}$ concentration during the last glacial period; Science 292 2453-2458

Blanchon P and Shaw J 1995 Reef-drowning during the last deglaciation: evidence for catastrophic sea level rise and ice sheet collapse; Geology $\mathbf{2 3}$ 4-8

Borole D V, Rajagopalan G and Somayajulu B L K 1987 Radiometric ages of phosphorites off the west coast of India; Marine Geology 78 161-165

Bosence D W J 1985 The morphology and ecology of a mound-building coralline alga (Neogonialithon strictum) from the Florida Keys; Palaentology 28 189-206

Carballo J D, Land L S and Miser D E 1987 Holocene dolomitization of supratidal sediments by active tidal pumping, Sugarloaf Key, Florida; J. Sediment. Petrol. 57 $153-165$

Done T J 1982 Patterns in the distribution of coral communities across the central Great Barrier Reef; Coral Reefs 1 95-107

Davies P J and Montaggioni L 1985 Reef growth and sea level change: the environmental signature; Proc. of the $5^{\text {th }}$ International Coral Reef Congress, Tahiti 3 477-515

Dullo W Ch, Camoini G F, Blomeier D, Colonna M, Eisenhauer A, Faure G, Casanova J and Thomassin B A 1998 Morphology and sediments of the fore-slopes of Mayotte, Comoro Islands: direct observations from a submersible. Spec. Pubs. Int. Ass. Sediment. 25 219-236

Dutta K, Bhushan R and Somayajulu B L K 2001. $\Delta$ R correction values for the northern Indian Ocean; Radiocarbon $43483-488$

Fairbanks R G 1989 A 17,000 year glacio-eustatic sea level record: influence of glacial melting rates on the Younger Dryas event and deep-ocean circulation; Nature $342637-$ 642

Hashimi N H, Nigam R, Nair R R and Rajagopalan G 1995 Holocene sea level fluctuations on western Indian continental margin: An update; J. Geol. Soc. India 46 157-162

Hallock P and Schlager W 1986 Nutrient excess and the demise of coral reefs and carbonate platforms; Palaios 1 389-398 
Hottinger L 1983 Neritic macroid genesis, an ecological approach; In: Coated grains (ed) Y Peryt (New York: Springer) p. $38-57$

Illings L V 1954 Bahamian calcareous sands; AAPG Bull. 38 1-95

Kale V S and Rajaguru S N 1985 Neogene and Quaternary transgressional and regressional history of the west coast of India - An overview; Bull Deccan College Res Inst. 44 153-165

Kump L R and Hine A C 1986 Ooids as sea level indicators; In: Sea level Research: A manual for the collection and evaluation of data (ed) O Van de Plasche (Geobooks Norwich) p. 175-194

Labeyrie L D, Duplessy J C and Blanc P L 1987 Variations in mode of formation and temperature of oceanic deep waters over the past 125,000 years; Nature 327 477-482

Logan B W, Harding J L, Ahr W M, Williams J D and Snead R G 1969 Carbonate sediments and reefs, Yucatan Shelf, Mexico; AAPG Memoir 11 1-98

Loreau J P and Purser G H 1993 Distribution of ultrastructure of Holocene ooids in the Persian Gulf; In: The Persian Gulf (ed.) G H Purser (New York: Springer-Verlag) p. $279-328$

Meldhal K H and Cutler A H 1992 Neotectonics and Taphonomy: Pleistocene Molluscan shell accumulations in the northern Gulf of California; Palaios 7 187-197

Merrill A S, Emery K O and Rubin M 1965 Ancient oyster shells on the Atlantic continental shelf; Science 147398 400

Minnery G A 1990 Crustose coralline algae from the Flower Garden Banks, Northwestern Gulf of Mexico: Controls on distribution and growth morphology; J. Sediment. Petrol. 60 992-1007

Montaggioni L F 2000 Post-glacial reef growth; $C R$ Acad Sci. Paris Sciences de la Terre et des Planets 331 319330

Nair R R 1974 Holocene sea levels on the western continental shelf of India; Proc. Indian Acad. Sci. 79B 197-203

Nair R R 1975 Nature and origin of small scale topographic prominences on the western continental shelf of India; Indian J. Mar. Sci. 4 25-29

Nair R R and Hashimi N H 1980 Holocene climatic inferences from the sediments of the western Indian continental shelf; Proc. Indian Acad Sci. Sec. B 89 229315

Nair R R and Pylee A 1968 Size distribution and carbonate content of the sediments of the western shelf of India; Bull. Nat. Inst. Sci. India 38 411-420

Nair R R, Hashimi N H and Gupta M V S 1979 Holocene limestones of part of the western continental shelf of India; J. Geol. Soc. India 20 17-23

Parkash B, Kumar S, Rao M S, Giri S C, Kumar C S, Gupta S and Srivastava P 2000 Holocene tectonic movements and stress fields in the western Gangetic plains; Curr. Sci. 79 438-449

Patterson R J and Kinsman D J 1982 Formation of diagenetic dolomite in coastal sabkha along Persian Gulf; AAPG Bull. 66 28-43

Plaziat J-Cl and Perrin Ch 1992 Multikilometer-sized reefs built by foraminifera (Solenomeris) from the early Eocene of the Pyrenean domain (S. France, N. Spain): Palaeo ecologic relations with coral reefs; Palaeogeogr. Palaeoclimat. Palaeoecol. 96 195-231

Rajendran K, Rajendran C P, Thakkar M and Tuttle M P 2001 The 2001 Kutch (Bhuj) earthquake: Coseismic surface features and their significance; Curr. Sci. 80 13971405

Rao K L 1975 India's water wealth (New Delhi: Orient Longman) $255 \mathrm{pp}$.
Rao S R 1996 From Dwaraka to Kurukshetra; J. Marine Archaeol. 5/6 61-71

Rao V P and Lamboy M 1996 Genesis of apatite in the phosphatized limestones of the western continental shelf of India; Marine Geology 136 41-53

Rao V P and Wagle B G 1997 Geomorphology and surficial geology of the western continental shelf and upper slope of India: A review; Curr. Sci. 73 330-350

Rao V P, Veerayya M, Nair R R, Dupeuble P A and Lamboy M 1994 Late Quaternary Halimeda bioherms and aragonitic faecal pellet-dominated sediments on the carbonate platform 'of the western continental shelf of India; Marine Geology 121 293-315

Rao V P and Veerayya M 1996 Submarine terrace limestones from the continental slope off Saurashtra-Bombay: Evidence of late Quaternary neotectonic activity; Curr. Sci. $7136-41$

Rao V P, Veerayya M, Thamban M and Wagle B G 1996 Evidences of late Quaternary neotectonic acitivity and sea level changes along the western continental margin of India, Curr. Sci. 71 213-219

Rao V P, Montaggioni L, Vora K H, Almeida F and Rao K M 2002a Significance of the carbonate reefs of the southwestern margin of India; Sediment. Geol. (in press)

Rao V P, Kessarkar P, Krumbein W E, Krajewski K P and Schneider R J 2002b Microbial Dolomite crust from the carbonate platform of the western margin of India; Sedimentology, (in press).

Reid R P and Macintyre I G 1988 Foraminiferal-algal nodules from the eastern Carribian: Growth history and implications on the value of nodules of palaeoenvironmental indicators; Palaios 3 424-436

Sarkar A, Ramesh R, Somayajulu B L K, Agnihotri R, Jull A $\mathrm{J}$ T and Burr G S 2000 High resolution Holocene monsoon record from the eastern Arabian Sea; Earth and Planetary Science Letters 177 209-218

Stuiver M, Reimer P J, Bard E, Beck J W, Burr G S, Hughen K A, Kromer B, McCormac F G, van der Plicht J and Spurkm M 1998. INTCAL 98 radiocarbon age calibration, 24,000-0 cal BP. Radiocarbon 40 1041-1083

Taberner C and Bosence D W J 1985 Ecological succession from corals to coralline algae in Eocene Patch reefs, Northern Spain; In: Paleoalgology: Contemporary Research and Applications (eds) D F Toomey and M H Nitecki (Berlin: Springer-Verlag), pp. 226-236

Thamban M, Rao V P, Schneider R R and Grootes P M 2001 Glacial to Holocene fluctuations in hydrography and productivity along the southwestern continental margin of India; Palaeogeogr. Palaeoclimat. Palaeoecol. 165 113127

Van Campo E 1986 Monsoon fluctuations in two 20,000 yr BP: Oxygen isotope records off southwest India; Quat. Res. 26 376-388

Veron J E N 1986 Corals of Australia and the Indo-Pacific, (London: Angus and Robertson) $644 \mathrm{pp}$.

von Stackelberg U V 1972 Faziesverteilung in sedimentes Indich-Pakistanischen Komtinental-Randes; (Arabisches Meer), Meteor Forschungsgebn, Reiche C.9 1-73

Vora K H and Almeida F 1990 Submerged reef systems on the central western continental shelf of India; Marine Geology 91 255-262

Vora K H, Wagle B G, Veerayya M, Almeida F and Karisiddaiah S M 1996 A 1300 km long late Pleistocene-Holocene shelf edge barrier reef system along the western continental shelf of India: occurrence and significance; Marine Geology 134 145-162

Vora K H, Gaur A S, Price D and Sundaresh 2002 Cultural sequence of Bet Dwarka island based on thermoluminescence dating; Curr. Sci. 82 1351-1356 
Wagle B G, Vora K H, Karisiddaiah S M, Veerayya M and Almeida F 1994 Holocene submarine terraces on the western continental shelf of India: Implications for sea level changes; Marine Geology 117 207225
Walter M R 1976 Stromatolites, Developments in Sedimentology 20790 pp. (Amsterdam: Elsevier)

Winland H D 1969 Stability of calcium carbonate polymorphs in warm shallow seawater; J. Sediment. Petrol. 39 1579-1587

MS received 22 April 2002; revised 30 September 2002 